PoS $\quad \begin{aligned} & \text { PROCEEDINGS } \\ & \text { OF SCIENCE }\end{aligned}$

\title{
A Brief Introduction to Particle Physics
}

\author{
Lian-Tao Wang* \\ University of Chicago \\ E-mail: liantaow@uchicago.edu
}

This is a brief introduction to particle physics based on my lecture at the TASI 2017 summer school.

Theoretical Advanced Study Institute Summer School 2017 "Physics at the Fundamental Frontier" 4 June - 1 July 2017

Boulder, Colorado

${ }^{*}$ Speaker. 


\section{Introduction}

This short note is based on the lecture I gave at the TASI 2017 summer school. The aim is to give an overview of particle physics. The targeted audience is graduate students who have no or little experience in working on particle physics. Instead of focusing on state of art detailed phenomenological studies in particle, I emphasized the basic structure and dynamics of the Standard Model. I have also included a brief introduction of the attempts at new physics beyond the Standard Model, with a focus on the electroweak symmetry breaking.

\section{The Standard Model}

\subsection{Our Starting Point}

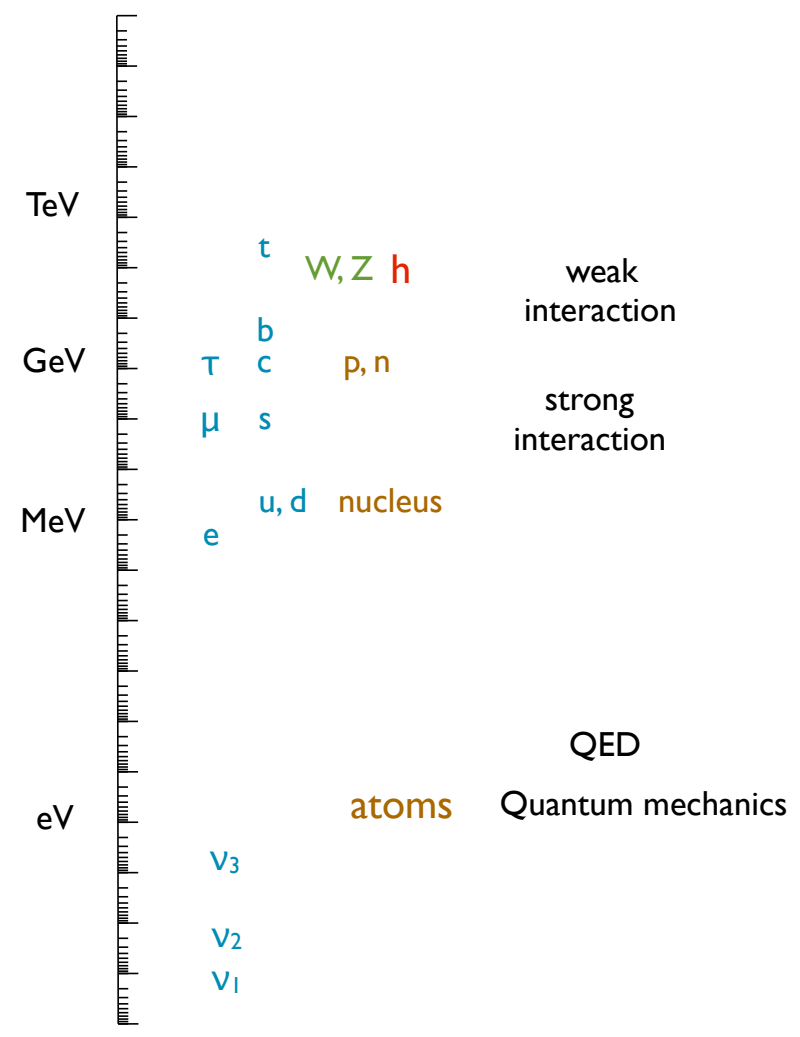

Figure 1: The scales in the Standard Model and relevant physics.

In this lecture, we regard the Standard Model as a low energy effective field theory (EFT), valid up to some high energy scale.

As an EFT, its basic ingredients are the particle content and the symmetries which govern their interactions. In a low energy EFT, the lowest order Lagrangian (for example, the renormalizable part of the Lagrangian) can have many so called accidental symmetries. Such symmetries play important roles in the leading order feature of the dynamics. It is the breaking of such accidental symmetries that reveals the presence of additional new physics. In general, the low energy effective 
theory can be written as

$$
\mathscr{L}=\mathscr{L}_{0}+\sum \frac{c_{i}}{M_{i}^{d-4}} \mathscr{O}^{(d)}
$$

$\mathscr{L}_{0}$ is the renormalizable part of the Lagrangian, sometime called (in some sense a misnomer) the Standard Model (SM). It has been very successful in predicting the results of all our current experimental data. The effects of new physics are parameterized by a set of so called higher dimensional operators $\mathscr{O}^{(d)}$, where $d>4$ is the cannonical dimension. By assumption, $\mathscr{O}$ are made from SM fields. $M_{i}$ is the scale of new physics, such as the masses of the new physics particles. Eq. 2.1 is the starting point of our dicussion of the Standard Model.

What if there are additional new particles beyond the Standard Model? In particular, some of these new particles may be as light as (or even lighter than) the SM particles. In this case, we obvious will need to include them into the low energy effective field theory. However, this also move us into a discussion of new physics beyond the Standard Model, which is beyond the scope of this section.

In the rest of the section, we will take a more detailed look at the SMEFT in Eq. 2.1. We will discuss what we know about the scale of new physics from current experimental observations. One might wonder if the SM could be the valid theory all the way to a very high scale, such as the quantum gravity scale $M_{\text {Planck }}$. This is indeed a possibility, and nothing we know tells us this is not so. However, this is an assumption we don't need to make. From the EFT point of view, it is sufficient to include all known degrees of freedom in the Lagrangian, and all possible (including non-renormalizable) interactions. Experiments should tell us the sizes of various terms in the EFT Lagrangian.

\subsection{Particle content and interaction}

\begin{tabular}{|c|c|c|c|} 
Particle & $S U(3)_{C}$ & $S U(2)_{L}$ & $U(1)_{Y}$ \\
\hline$Q$ & 3 & 2 & $\frac{1}{6}$ \\
$u^{c}$ & $\overline{3}$ & 1 & $-\frac{2}{3}$ \\
$d^{c}$ & $\overline{3}$ & 1 & $\frac{1}{3}$ \\
$L$ & 1 & 2 & $-\frac{1}{2}$ \\
$e^{c}$ & 1 & 1 & 1 \\
$H$ & 1 & 2 & $\frac{1}{2}$ \\
$G_{\mu}$ & 8 & 1 & 0 \\
$W_{\mu}$ & 1 & 3 & 0 \\
$B_{\mu}$ & 1 & 1 & 0
\end{tabular}

Table 1: The particle content and the gauge interactions of the SM Model. The first six rows are the so called matter fields (not an accurate name), while the last three rows are the gauge bosons corresponding to the three gauge interactions of the Standard Model. We are using the convention that only left-handed fermion fields are presented here. There are three generations of quarks and leptons (only one generic generation shown here).

Table 1 summarizes the particle content and the gauge interactions of the Standard Model [1]. This is shown in the so called gauge eigenstate basis, which is more convenient in discussing 
physics at high energies. Around $10^{2} \mathrm{GeV}$, the so called electroweak symmetry is spontaneously broken, $S U(2)_{L} \times U(1)_{Y} \rightarrow U(1)_{\mathrm{EM}}$, a phenomenon we will discuss in detail later. After that, many particles acquire masses and it is more convenient to go to a mass eigenstate basis. Going to lower energies, another important event happens at around a $\mathrm{GeV}$, in which the strong interaction becomes strong, and quarks and gluons stop being relevant degrees of freedom. We will also describe the resulting theory in detail later in this section.

\subsubsection{Electroweak symmetry breaking}

It is well known that spontaneous symmetry breaking and the Higgs mechanism [2] are responsible for the dynamics of the SM electroweak interactions. Of course, a major milestone of the story is the discovery of the Higgs boson in 2012. Here, we will first follow a more "historical" approach to appreciate the importance of this discovery.

After the discovery of $W$ and $Z$ gauge bosons in early 80 's, we had to include in our particle physics Lagrangian the masses of these spin-1 gauge bosons,

$$
\mathscr{L} \supset m_{W}^{2} W^{+} W^{-}+\frac{1}{2} m_{Z}^{2} Z^{2} .
$$

It is often said that these terms are not gauge invariant. This is not quite right. To simplify our discussion, we will see how this works on a generic vector boson $A_{\mu}$. We will make the following change to its mass term

$$
m^{2} A_{\mu}^{2} \rightarrow m^{2}\left(A_{\mu}-\frac{1}{m} \partial_{\mu} \pi(x)\right)^{2} .
$$

These two forms of the mass term are equivalent since they are related by a simple field redefinition $A \rightarrow A(x)-1 / m \partial \pi(x)$. However, in the second form, the mass term is obviously gauge invariant with the following gauge transformation

$$
A_{\mu}(x) \rightarrow A_{\mu}+\partial_{\mu} \alpha(x), \pi(x) \rightarrow \pi(x)+m \alpha(x) .
$$

Therefore, strictly speaking, adding mass terms for gauge bosons does not break the so called gauge invariance. It does have a physical effect, which is adding a new dynamical degree of freedom. The mass term now gives a kinetic term to the new $\pi(x)$ field, i.e., it propagates the new degree of freedom. This is exactly what we needed for having a massive spin-1 particle. After all, the little group analysis tells us that a massive spin-1 particle will have 3 degrees of freedom. In this way, we also see "just adding a mass term", shown in Eq. 2.2, just corresponds to writing the theory in the unitary gauge, where $\pi(x)$ is implicit. The $\pi(x)$ field is nothing but the eaten Goldstone (sometimes called would be Goldstone), and what we have discussed here is the Higgs mechanism.

Now, the Higgs boson discovered in 2012 at the LHC [3] is a neutral scalar, and it is not part of a gauge boson. As we have seen in the previous paragraph, it is not really needed to give the gauge boson mass. Therefore, we should ask what it does in the Standard Model. In fact, far from just being an extra particle, it plays a extremely important role. To understand this, let's go back the case of a theory with just massive spin-1 gauge bosons, a.k.a. the Standard Model before 2012. Let's consider the following scattering process

$$
W+W \rightarrow W+W .
$$




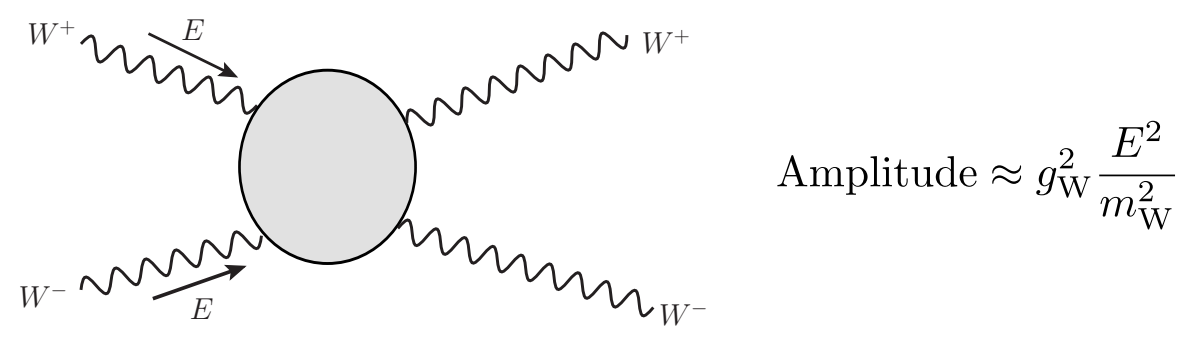

Figure 2: Scattering of $\mathrm{W}$ bosons. Its amplitude grows as $E^{2}$. This is an indication of presence of new physics below a TeV.

In particular, let's focus on scatterings at high energies $E>m_{W}$. For the three polarizations of the $\mathrm{W}$ boson, we expect the longitudinal polarized $\mathrm{W}$ boson to dominate the scattering amplitude. This can be seen in the unitary gauge. The longtudinal polarization vector is

$$
\varepsilon_{\mu}^{L} \sim p_{\mu} / m_{W}+\mathscr{O}\left(m_{W} / E\right)
$$

which grows with energy. At the same time, the transverse polarizations do not grow with energy. In the gauge where the Goldstone is manifest, the scattering of these Goldstones, i.e. the would be longitudinal modes, would be dominant at high energies. In fact, according to the Goldstone Equivalence Theorem [4], at high energies, the scattering amplitude for the longitudinal modes is the same as the scattering of the Goldstones. Therefore, we can understand this amplitude by just considering the non-linear sigma model of the Goldstones. To this end, we define

$$
\Sigma=\exp \left(i \frac{2 \pi(x)}{v}\right), \pi(x)=t^{a} \pi^{a}(x)
$$

$t^{a}$ S are the corresponding generators, such as the $S U(2)_{L}$ generators $t^{a}=\sigma^{a} / 2$ for the SM weak interaction. The leading order Lagrangian is

$$
\mathscr{L}=\frac{v^{2}}{4} \operatorname{tr}\left[\partial_{\mu} \Sigma \partial^{\mu} \Sigma\right]
$$

Expand the exponential beyond the usual kinetic term for $\pi^{a}$, we have

$$
\mathscr{L} \supset \frac{1}{v^{2}}\left[\left(\pi^{a} \partial_{\mu} \pi^{a}\right)^{2}-\left(\pi^{a} \partial_{\mu} \pi^{b}\right)^{2}\right] .
$$

From this, we can conclude the scattering amplitude for $\pi \pi$ scattering grows with energy as

$$
\mathscr{M}(\pi+\pi \rightarrow \pi+\pi) \sim \frac{E^{2}}{v^{2}},
$$

due to the presence of derivatives in their interaction. A careful computation of scattering of the longitudinal modes of Ws yields the result, as required by the Goldstone Equivalence Theorem. We also note that this is a phenomenon special to non-Abelian gauge interactions. For an Abelian gauge interaction, there is only one would be Goldstone, and the interaction in Eq. 2.9 vanishes. 
We can view this amplitude as a measurement of the strength of a 4-Goldstone interaction. Eq. 2.10 shows that this coupling is growing with energy $\propto E^{2}$. This means that this theory with massive gauge bosons can not be a valid description at arbitrarily high energies. At some point, the theory will become strongly coupled, or "violate unitarity" in some literature, and it would need to be UV completed by some new physics. In particular, somewhat arbitrarily requiring the coupling less than $(4 \pi)^{2}$, we expect the new physics to set in below

$$
\Lambda_{\mathrm{NP}}<4 \pi v=\frac{4 \pi m_{W}}{g} \sim \mathrm{TeV} .
$$

This is a very important conclusion. We knew, after the discovery of the $W^{ \pm}$and $Z$, that the theory can not be complete and there must be new physics below about a TeV [5].

What could this new physics be? There are obviously many possibilities. For example, an elaborate model would be that all the would be Goldstones are composite particles formed by the confinement of a new strong interaction around $\mathrm{TeV}$ scale. In this picture, the UV completion would be that the would be Goldstones stop being fundamental degrees of freedom around TeV. This may seem to be a stretch. However, we should note that this is exactly what happens to the pion pion scattering in QCD. A scenario along this lines is called technicolor [6], or its more modern reincarnation, the Higgsless models. Despite common impression and some uneasiness with experimental constraints (we will review some of them later), they remained viable models before 2012 .

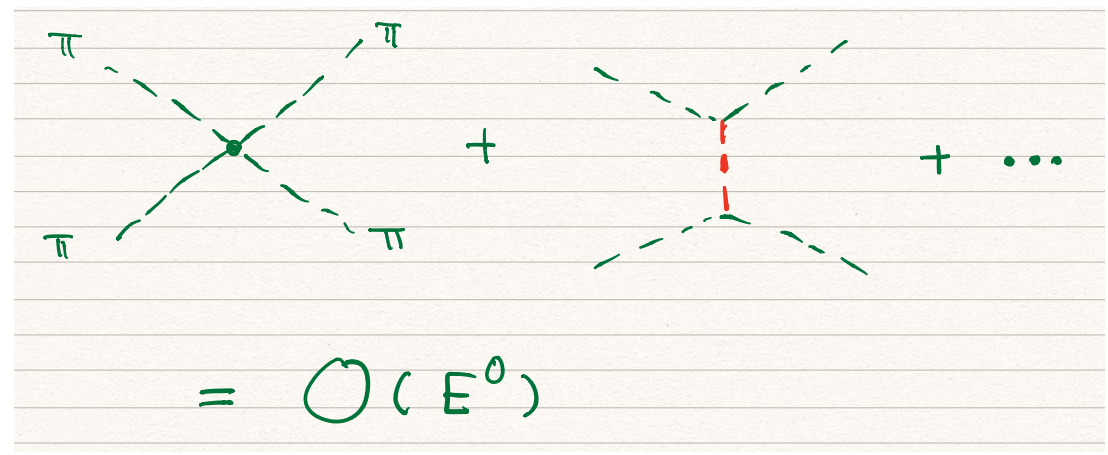

Figure 3: Higgs unitarizes the WW scattering amplitude. The longitudinal modes of $\mathrm{W}$ are replaced by respective Goldstones $\pi$, and the Higgs boson exchange is shown with red dashed line.

At the same time, there is a much simpler UV completion which only involves one new degree of freedom. We could extend the $\Sigma$ field by

$$
\Sigma \rightarrow \Phi=\frac{1}{\sqrt{2}}(v+\hat{h}) e^{i 2 \pi / v}
$$

where we have include one real scalar field $\hat{h}$. First, let's notice that this is a good UV completion, in the sense that it removes energy growing behavior of the $\pi \pi$ scattering amplitude, and renders the theory weakly coupled at high energies. This can be seen by noticing that the new field $\Phi$ is just a reparamterization of another field

$$
\Phi \leftrightarrow H=\frac{1}{\sqrt{2}}\left(\begin{array}{c}
a_{1}+i a_{2} \\
v+h+i a_{0}
\end{array}\right) .
$$


On the other hand, the theory for the doublet $H$ is just the usual linear $\sigma$-model with

$$
\mathscr{L} \supset\left|D_{\mu} H\right|^{2},
$$

which obviously does not have any bad UV behavior. The single new degree of freedom we have added, a real neutral scalar, is the so called Higgs boson. It plays the role of UV completing the SM, which then can be valid up to much higher energies. As we have remarked, this is perhaps the simplest UV completion. However, being simple does not always imply it is the right UV completion. In fact, there are concerns about having a light elementary scalar particle which we will discuss in detail later. Therefore, the importance of the discovery of a Higgs boson by the ATLAS and CMS collaborations [3] is to verify this is indeed the UV completion of the SM massive gauge bosons. Since the experiment evidence so far all point towards the interaction of the form shown in Eq. 2.14, it has become the "standard" to test. Therefore, we will adopt the common usage and call it the Standard Model from now on.

We should emphasize again that the Higgs boson itself, in contrast to many popular account, is not the origin of mass. It is not even the one breaking the electroweak symmetry. In fact, it is a total SM singlet. The physical definition of the Higgs boson is that its contribution to the vector boson scattering amplitude cancels the potential bad high energy behavior. In order to play this role, the couplings of the SM Higgs boson, in particular those to the SM gauge bosons, must be fixed to particular values. In particular, we can write

$$
\frac{m_{W}^{2}}{v}\left(a h+\frac{b}{v} h^{2}+\ldots\right) \times W^{+} W^{-} .
$$

The SM predicts $a=2$ and $b=1$, which are consistent with the experimental measurements so far. It is up to the upcoming experiment to further test these predictions.

\subsubsection{Strong Interaction}

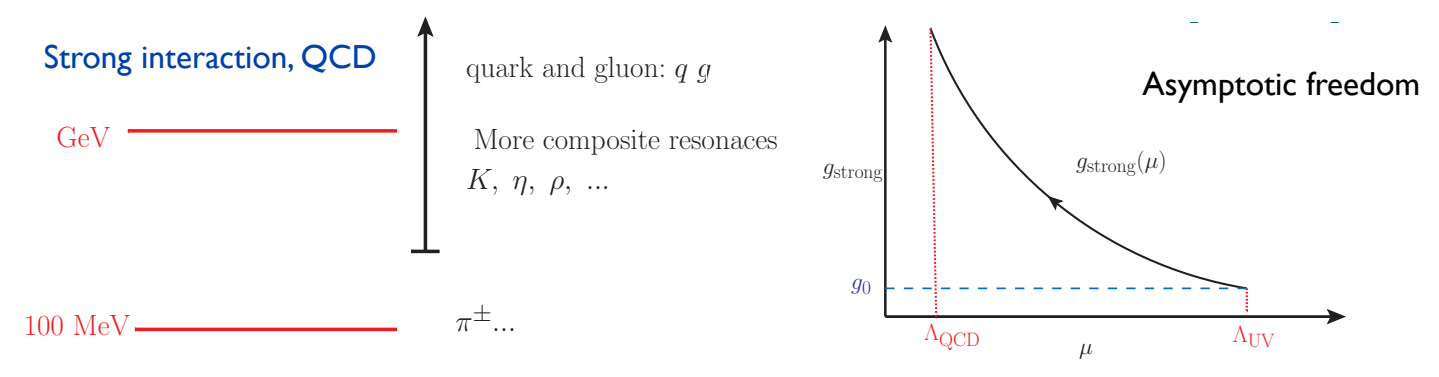

Figure 4: QCD, theory of strong interaction.

The theory of the strong interaction of the Standard Model, called Quantum Chromodynamics (QCD), offers a different array of extremely interesting physics phenomena.

The most prominent feature of the QCD of the Standard Model is the so called asymptotic freedom [7]. At high energies, or short distances, the theory is weakly coupled. Hence, it can be roughly thought of as free gluons and quarks. At the same time, at low energies $\sim 1 \mathrm{GeV}$, the gauge coupling becomes strong and the theory become strongly coupled. QCD enters confinement 
phase, where the relevant degrees of freedom are many hadronic bound states. While we can use standard perturbation theory techniques for the high energy process involving quarks and gluons, our capability of making precise calculation in the strongly coupled regime is limited.

One of the important stages for the QCD physics is high energy collisions in which either the incoming or out-going states (or both) are hadronic. QCD can also give important contribution to virtual corrections. These processes involve both perturbative and non-perturbative aspects of QCD. The structure of the incoming protons at hadron colliders and the formation of hadronic resonances as out going particles are examples of such incalculable quantities. Given these, it is a remarkable feat that the experimental outcomes at colliders, in particular at hadron colliders, are actually predictable by using a combination of theoretical calculation and experimental inputs. This is primarily due to a phenomenon called factorization. The basic idea of factorization is simple, and it makes a lot of sense. It is based on one of the fundamental concepts of modern quantum field theory, namely, the physics at different length scales only influence each other in controllable ways. For example, this is the foundation for the effective field theory framework. In the context of QCD, factorization in high energy collisions means that the short-distance process ( called hard collision and is computable in perturbation theory) and long distance physics enter the final results as separate factors. Intuitively, this is due to the fact that long-distance physics, happening over a longer time scale, can not influence the detail of the hard collision. Schematically, at hadron colliders, the differential cross section of some observable $d \sigma$ can be written as

$$
d \sigma=P_{1} \otimes P_{2} \otimes d \hat{\sigma} J_{1} \otimes J_{2} \ldots \otimes S .
$$

Symbol $\otimes$ means the result is a convolution rather than a simple product. $P_{1,2}$ are the so called beam functions, which summarize the properties of the incoming beams. Approximately, these are the so called parton distribution functions. They can not be computed from first principles (yet), and have to be measured from experiments. The key implication of factorization is that the effect of the structure of the incoming hadrons (typically protons) can be summarized in these universal functions. Once they are measured in one set of experiments, they can be used to make predictions for others. $S$ are some soft function which can not be computed perturbatively either. Typically, one can design experimental observable to be as insensitive to $S$ as possible. $J_{i}$ s are the so called the jet functions, named after perhaps the most commonly used object in characterizing hadronic activities. These are determined by a combination of perturbative resummation technique and nonperturbative information. Making good predictions for hadron collider observables is a deep and complicated subject. The picture presented above is no doubt too simple. Delving deeper into it will not be the focus of this set of lectures [8]. We make one last remark about factorization. As simple as it sounds, it is extremely difficult to prove in full generality especially for hadron collider processes, even though proofs do exist for some simple processes. In practice, we will have to make the assumption that the process (and the associated observable) do factorize before we can make any progress. Fortunately, we can then compare the result we obtain with the experiment, and in almost all cases, they do agree. There have been a large collection of amazing success in using QCD to predict observables at high energy colliders, such as the Large Hadron Collider.

Next, we move to the low energy regime of QCD. Since the QCD, as a theory of gluons and quarks, becomes strongly coupled at around the QCD scale $\Lambda_{\mathrm{QCD}} \sim \mathrm{GeV}$, we can not use perturbation theory to directly derive observables for experiment conducted below the QCD scale from the 
interactions of gluons and quarks. From the effective theory point of view, the low energy degrees of freedom of QCD are a collection of resonances. Therefore, we should aim at developing an effective field theory for these resonances. We start with global symmetries. Here, we are inspired by the high energy QCD. As a vector like theory with $N_{f}$ flavors, if we ignore the quark masses, it has an $S U\left(N_{f}\right)_{L} \times S U\left(N_{f}\right)_{R}$ chiral global symmetry. Quark masses break these symmetries. However, as usual in physics, we can still make progress if we could find approximate symmetries with small breaking parameters which we can treat as perturbations. For QCD, we note that at least two quarks, up and down, are very light. More specifically, $m_{u, d} \ll \Lambda_{\mathrm{QCD}}$. Therefore, if we ignore their masses, we can approximately regard QCD as having a $S U(2)_{L} \times S U(2)_{R}$ symmetry. In practice, it is often a good approximation to also treat the strange quark as light. In this case, we begin with a $S U(3)_{L} \times S U(3)_{R}$ symmetry. What happens to these symmetries at energies below QCD scale? For that, we note that there are a set of light scalars, $\pi^{ \pm}, \pi^{0}, K^{0}$, etc., with masses quite a bit below the QCD scale. Since it is not generic for scalars to be accidentally light, it would be a natural guess that these are approximately Nambu-Goldstone bosons, resulting from the breaking of global symmetries. It turns out that for QCD, there is an powerful argument from anomaly matching [9] that the high energy chiral symmetries have to be spontaneously broken.

First, we start with the global symmetry breaking

$$
S U(3)_{L} \times S U(3)_{R} \rightarrow S U(3)_{V}
$$

Goldstone theorem [10] tells us that there would be 8 Goldstones, which we denote as $\pi^{a}, a=$ $1,2, \ldots, 8$, associated with 8 broken generators $T^{a}, a=1,2, \ldots 8$. There are some different options to parameterize the Goldstones. We will begin with defining the non-linear $\sigma$-model field

$$
U(x)=\exp \left(\frac{2 i}{f_{\pi}} \hat{\pi}(x)\right), \hat{\pi}=\Sigma_{a} \pi^{a}(x) T^{a} .
$$

$f_{\pi}$ is a parameter to be determined by experiment. These Goldstones are mesons observed expermentally, and they also have more "historical" names [11]

$$
\hat{\pi}=\frac{1}{\sqrt{2}}\left(\begin{array}{ccc}
\frac{1}{\sqrt{2}} \pi^{0}+\frac{1}{\sqrt{6}} \eta & \pi^{+} & K^{+} \\
\pi^{-} & -\frac{1}{\sqrt{2}} \pi^{0}+\frac{1}{\sqrt{6}} \eta & K^{0} \\
K^{-} & \bar{K}^{0} & \frac{-2}{\sqrt{6}} \eta
\end{array}\right) .
$$

Under the chiral symmetries,

$$
S U(3)_{L} \times S U(3)_{R}: U \rightarrow V_{L} U V_{R}^{\dagger} .
$$

The VEV $\langle U\rangle=\mathbf{1}$ breaks the chiral symmetries to the diagonal $S U(3)$. The leading order $S U(3)_{L} \times$ $S U(3)_{R}$ invariant Lagrangian is

$$
\mathscr{L}_{0}=\frac{f_{\pi}^{2}}{4} \operatorname{tr}\left(\partial_{\mu} U \partial^{\mu} U^{\dagger}\right) .
$$

We are using the convention $\operatorname{tr}\left(T^{a} T^{b}\right)=\frac{1}{2} \delta^{a b} . U$ transforms under infinitesmal chiral rotation, for example

$$
U \rightarrow U+i \varepsilon^{a} T^{a} U
$$


Using these, we can derive the Noether currents

$$
j_{L}^{a \mu}=i \frac{f_{\pi}^{2}}{2} \operatorname{tr}\left(T^{a} U \partial^{\mu} U^{\dagger}\right), j_{R}^{a \mu}=i \frac{f_{\pi}^{2}}{2} \operatorname{tr}\left(T^{a} U^{\dagger} \partial^{\mu} U\right) .
$$

Or sometimes more usefully, we can form the combination

$$
j_{5}^{a \mu}=j_{R}^{a \mu}-j_{L}^{a \mu}=-f_{\pi} \partial^{\mu} \pi^{a}+\mathscr{O}\left(\pi^{2}\right) .
$$

From this, we can write

$$
\left\langle 0\left|j_{5}^{a \mu}(x)\right| \pi^{b}(p)\right\rangle=i p^{\mu} f_{\pi} \delta^{a b} e^{-i p \cdot x} .
$$

Together with electroweak interaction of the SM, we can then compute the pion decay partial width for $\pi^{+} \rightarrow \mu^{+}+v$

$$
\Gamma\left(\pi^{+} \rightarrow \mu^{+} v\right)=\frac{G_{F}^{2} f_{\pi}^{2}}{4 \pi} m_{\pi} m_{\mu}^{2}\left(1-\frac{m_{\mu}^{2}}{m_{\pi}^{2}}\right)^{2} .
$$

Experimental measurement of muon decay then fixes $f_{\pi}=92 \mathrm{MeV}$.

We still have to add the quark masses. In flavor space, the quark masses can be collected in a $3 \times 3$ matrix

$$
M=\left(\begin{array}{lll}
m_{u} & & \\
& m_{d} & \\
& & m_{s}
\end{array}\right) .
$$

Next, we need to add it to the Lagrangian of pions. To proceed, we note that the chiral symmetry can be restored in the QCD Lagrangian if we consider the quark mass matrix transforms under chiral symmetries

$$
S U(3)_{L} \times S U(3)_{R}: \quad M \rightarrow V_{L} M V_{R}^{\dagger} .
$$

Then, a standard spurion analysis tells us the proper mass terms in the chiral Lagrangian should be

$$
\mathscr{L}_{\text {meson mass }}=a_{m} \Lambda_{\mathrm{QCD}}^{3} \operatorname{tr}\left(U^{\dagger} M+M^{\dagger} U\right)+\mathscr{O}\left(M^{2}\right) .
$$

Expand this expression out we obtain meson masses

$$
\begin{aligned}
m_{\pi^{0}}^{2} & =\frac{a_{m} \Lambda_{\mathrm{QCD}}^{3}}{f_{\pi}^{2}}\left(m_{u}+m_{d}\right)+\mathscr{O}\left(m_{u, d} / m_{s}\right), \\
m_{\pi^{ \pm}}^{2} & =\frac{a_{m} \Lambda_{\mathrm{QCD}}^{3}}{f_{\pi}^{2}}\left(m_{u}+m_{d}\right)+\delta m_{\mathrm{QED}}^{2}, \\
m_{K^{0}, \bar{K}^{0}}^{2} & =\frac{a_{m} \Lambda_{\mathrm{QCD}}^{3}}{f_{\pi}^{2}}\left(m_{d}+m_{s}\right), \\
m_{K^{ \pm}}^{2} & =\frac{a_{m} \Lambda_{\mathrm{QCD}}^{3}}{f_{\pi}^{2}}\left(m_{d}+m_{s}\right)+\delta m_{\mathrm{QED}}^{2}, \\
m_{\eta}^{2} & =\frac{1}{3} \frac{a_{m} \Lambda_{\mathrm{QCD}}^{3}}{f_{\pi}^{2}}\left(m_{u}+m_{d}+m_{s}\right) .
\end{aligned}
$$

These Gell-Mann-Okubo relations [11] are in good agreement with the experimental data [12], given the level of the approximation. The QED contributions $\delta m_{\mathrm{QED}}^{2}$ are calculable. 
We have so far introduced two set of terms in the chiral Lagrangian $\mathscr{L}_{\text {chiral }}=\mathscr{L}_{0}+\mathscr{L}_{\text {meson mass }}$. In addition to meson masses and some electroweak decays, it can also describe processes such as $\pi \pi \rightarrow \pi \pi$ scattering. The perturbation is in the powers of $E / \Lambda_{\mathrm{QCD}}$ and $m / \Lambda_{\mathrm{QCD}}$. We can certainly push this program further, including more terms in the chiral Lagrangian and going beyond tree level. This is the so called chiral perturbation theory. In these lectures, we will not go to further details of this rich field.

There is one more class of terms in the chiral Lagrangian we should discuss. The chiral Lagrangian we have discussed so far has more discrete symmetries than the QCD Lagrangian. It has a naive parity $P_{0}: U(t, \mathbf{x}) \rightarrow U(t,-\mathbf{x})$ and a pion number $(-1)^{N_{\pi}}$ symmetry. In the original $\mathrm{QCD}$, on the other hand, has only the product of these symmetries. As a result, processes such as $K^{+} K^{-} \rightarrow \pi^{+} \pi^{-} \pi^{0}$, which are allowed in QCD, would not be allowed by the chiral Lagrangian we have written down so far. Additional interactions are needed. Witten identified such terms [13], which has been derived earlier by Wess and Zumino by studying the chiral anomalies [14]. We will not repeat the discussion here. Instead, we will describe some physics consequences of these so called Wess-Zumino-Witten terms. As discussed above, it can describe the processes in which even number of Goldstones go to odd number of Goldstones, such as $K^{+} K^{-} \rightarrow \pi^{+} \pi^{-} \pi^{0}$. We can also gauge part of the chiral symmetry, for example, the SM gauge group. Perhaps the most famous case is the gauging of $U(1)_{\mathrm{EM}}$. This leads to a coupling

$$
\mathscr{L} \supset \frac{n e^{2}}{96 \pi^{2}} \frac{1}{f_{\pi}} \pi^{0} F \tilde{F}
$$

An argument similar to the Dirac quantization leads to the conclusion that $n$ needs to be an integer. Anomaly matching in QCD fixes it to be the same as the number of colors $n=N_{C}=3$. Historically, the measurement of decay $\pi^{0} \rightarrow \gamma \gamma$ is the one of the earliest observations which allowed us to "count" the number of colors of QCD. Similarly, sucextension of the chiral Langrangian with WZW terms also allows interactions such as $\gamma \pi^{+} \pi^{-} \pi^{0}$.

\subsection{Accidental symmetries}

The Standard Model have (approximate) global symmetries. From an effective field theory point of view, such symmetries should be viewed as accidental, i.e., they are expected to be broken by higher dimension operators not present in the renormalizable Lagrangian. These symmetries (and their potential breaking at higher energies) prodive important organizing principles for thinking about new physics beyond the Standard Model. In this section, we offer a brief overview of such symmetries.

\subsubsection{Custodial $S U(2)$ symmetry}

Rewrite the SM Higgs doublet as

$$
H=\left(\begin{array}{c}
h^{+} \\
h^{0}
\end{array}\right) \rightarrow \Phi=\left(\begin{array}{cc}
h^{0 *} & h^{+} \\
h^{-} & h^{0}
\end{array}\right) .
$$

The kinetic terms of the Higgs can be written as

$$
\mathscr{L} \supset\left(D_{\mu} H^{\dagger}\right)\left(D^{\mu} H\right)=\operatorname{tr}\left(\left(D^{\mu} \Phi\right)^{\dagger} D_{\mu} \Phi\right),
$$


where

$$
D_{\mu} \Phi=\partial_{\mu} \Phi+i g \frac{\vec{\sigma}}{2} \cdot \vec{W}_{\mu} \Phi-i \frac{g^{\prime}}{2} B_{\mu} \Phi \sigma_{3} .
$$

The Lagrangian is obvious invariant under $S U(2)_{L}$ rotations

$$
S U(2)_{L}: \Phi \rightarrow U_{L} \Phi, \frac{\vec{\sigma}}{2} \cdot \vec{W}_{\mu} \rightarrow U_{L} \frac{\vec{\sigma}}{2} \cdot \vec{W}_{\mu} U_{L}^{\dagger} \text {, hence, } D_{\mu} \rightarrow U_{L} D_{\mu} \Phi
$$

If we ignore the Hypercharge gauge interaction with $B_{\mu}$ (last term in the Lagrangian) for the moment, there is one more symmetry in the Lagrangian

$$
S U(2)_{R}: \Phi \rightarrow \Phi U_{R}^{\dagger}, D_{\mu} \Phi \rightarrow D_{\mu} \Phi U_{R}^{\dagger}
$$

After electroweak symmetry breaking,

$$
\Phi \rightarrow\langle\Phi\rangle=\left(\begin{array}{c}
v \\
v
\end{array}\right) .
$$

The symmetry breaks down to the diagonal

$$
S U(2)_{L} \times S U(2)_{R} \rightarrow S U(2)_{C} .
$$

$S U(2)_{C}$ is the so called Custodial Symmetry of the Standard Model [15]. Under the Custodial Symmetry, the $\mathrm{W}$ bosons transform as a triplet. This implies, at this step,

$$
m_{W^{ \pm}}=m_{W^{1}}=m_{W^{2}}=m_{W^{3}} .
$$

Of course, the Custodial Symmetry is not exact in the Standard Model, even with just renormalizable interactions. However, it is important that it is broken in a controllable and calculable way.

At tree level, the Custodial Symmetry is broken by a small parameter, it is the Hypercharge gauge interaction which we have ignored above. Turning on the Hypercharge, $W_{3}$ now mixes with the $B_{\mu}$ and produces two neutral states $Z^{0}$ and $\gamma \cdot m_{Z^{0}} \neq m_{W^{ \pm}}$, and their ratios is

$$
\rho=\frac{m_{W}^{2}}{m_{Z}^{2} \cos ^{2} \theta_{W}}=1, \text { with } \cos ^{2} \theta_{W}=\frac{g^{2}}{g^{2}+g^{\prime 2}}
$$

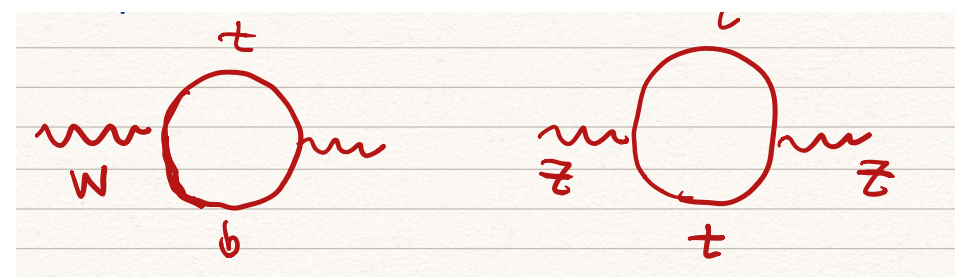

Figure 5: One loop contribution to $W$ and $Z$ masses from 3rd generation of quarks in the Standard Model.

There are additional Custodial Symmetry breakings in the Standard Model. The most important one of these is the Yukawa coupling of the 3rd generation quarks

$$
\frac{1}{\sqrt{2}}\left(t_{L}, b_{L}\right)\left(\begin{array}{c}
v \\
\\
v
\end{array}\right)\left(\begin{array}{cc}
y_{t} & \\
& y_{b}
\end{array}\right)\left(\begin{array}{c}
t_{R} \\
b_{R}
\end{array}\right),
$$


in which the breaking effect is proportional to $y_{t}-y_{b} \simeq y_{t}$. This would contribute to the mass relation of $W$ and $Z$ bosons at one-loop level

$$
\delta \rho \simeq \frac{3}{8 \sqrt{2} \pi^{2}} G_{F} m_{t}^{2} \sim 0.01
$$

Notice that it is proportional to top mass square. This is one of the early indirect indications of the range of the top mass.

The Hypercharge interaction also contributes to the mass relation at one-loop

$$
\delta \rho \simeq \frac{11}{24 \sqrt{2} \pi^{2}} g^{\prime 2} G_{F} \log \frac{m_{h}^{2}}{m_{Z}^{2}} .
$$

Notice that it depends on the Higgs mass logarithmically. Historically, this offers a first (albeit weak) indication of the range of the Higgs mass.

We can push further to include more corrections, including smaller effects and higher loops. This will lead to more precise predictions based on the renormalizable interactions of the SM, which can be compared with experiments. This physics program is called electroweak precision measurements. It has provided some of the most stringent test of the Standard Model [16].

As any accidental symmetry, we expect the Custodial Symmetry can be broken by higher dimensional operators. The leading order symmetry breaking operator is of dimension 6

$$
\mathscr{L} \supset c \frac{v^{2}}{M^{2}}\left[\operatorname{tr}\left(\sigma_{3} \Phi^{\dagger} D_{\mu} \Phi\right)\right]^{2},
$$

where $M$ is the mass of some new physics state which is responsible for breaking the Custodial Symmetry. The appearance of $\sigma_{3}$ is expected since it is the spurion which breaks the Custodial Symmetry. $c$ parameterizes the coupling of the new physics to the SM particles. At least, we expect $c$ to be not too much larger than $\mathscr{O}(1)$. Experimentally, we measured the mass relation to per mil precision

$$
\delta \rho<10^{-3}
$$

Then, with the assumption on the coupling

$$
c \sim \mathscr{O}(1) \rightarrow M>3 \mathrm{TeV} .
$$

\subsubsection{Baryon number and Lepton number}

We assign the Baryon number (B) and the Lepton number (L) of the SM matter fields as follows

\begin{tabular}{c|c|c|c} 
& $Q, u, d$ & $L, e$ & $H$ \\
\hline$B$ & $\frac{1}{3}$ & 0 & 0 \\
$L$ & 0 & 1 & 0
\end{tabular}

These are symmetries of the renormalizable interactions of the Lagrangian. They are broken by the electroweak anomalies down to the combination $B-L$. However, these effects are small $\sim$ $\exp \left(-1 / g^{2}\right)$. For most of our purposes, we can still think of $B$ and $L$ are separately conserved. The only except would be in the early universe when $T \geq m_{W}$. Baryon number violation are strongly 
constrained, especially if the lepton number symmetry is broken simultaneously. For example, dimension 6 operator

$$
\frac{1}{M^{2}} Q Q Q L
$$

leads to proton decay. This leads to a very stringent limit [12]

$$
\tau_{\text {proton }}>10^{33} \text { yrs } \rightarrow M>10^{15} \mathrm{GeV}
$$

The fate of the Lepton number symmetry is more subtle, since it is related to the origin of the neutrino mass, which is still an open question. With only the degrees of freedom in the Standard Model, the leading order operator which can break Lepton number symmtry is the Weinberg operator (dimension 5) [17]

$$
\frac{c_{v}}{M}(L H)^{2}
$$

This operator, after electroweak symmetry breaking, gives neutrino mass on the order of

$$
m_{v} \simeq c_{v} \frac{v^{2}}{M}
$$

This operator violates the Lepton number by 2 units. The resulting neutrino mass term is Majorana, in the sense that, with the mass term, there is no conserved Lepton number (or any other conserved quantum number ) to distinguish neutrino from anti-neutrino. Assuming $c_{v} \sim \mathscr{O}(1)$ and $m_{v} \sim 0.1$ $\mathrm{eV}$, we have $M \sim 10^{13} \mathrm{GeV}$.

A typical way of generating the Weinberg operator is to introduce a heavy singlet fermion $\mathrm{N}$, with

$$
\mathscr{L} \supset y_{v} L H N+\text { h.c. }+M N N \text {. }
$$

Integrating our the singlet generates the Weinberg operator with $c_{v}=\left|y_{v}\right|^{2}$. This is the so called (Type 1) see-saw mechanism [18]. It is an appealing way of generating the small neutrino mass since it explains a small number with a large mass hierarchy.

One could, of course, introduce a new light singlet $n$ with

$$
\mathscr{L} \supset y_{v} L H n+\text { h.c. }
$$

This term preserves the Lepton number with the assignment $L(n)=-1$. Hence, we can distinguish neutrino and anti-neutrino, and this is called a Dirac mass term. The neutrino mass is $m_{v}=(1 / \sqrt{2}) y_{v} v$, which implies we need a small Yukawa coupling to give the correct neutrino mass.

Perhaps most of the theorists prefers the see-saw mechanism. This is for the reason stated above. In addition, it is easy to make it into a Baryogenesis model with the leptogenesis mechanism [19]. However, only experiment can tell which one is correct. The most important measurement is obviously testing whether lepton number is violated by two units. Neutrinoless double beta decay has the best potential to probe this effect. 


\subsubsection{Flavor symmetries}

To begin with, we switch off all the Standard Model Yukawa interactions (hence fermion masses). The remaining part of the Standard Model Lagrangian has the following flavor symmetries

$$
G_{\text {flavor }}=U(3)_{Q} \times U(3)_{u} \times U(3)_{d} \times U(3)_{L} \times U(3)_{e} .
$$

In the Standard Model, $3 \times 3$ Yukawa matrices

$$
Y_{u}, Y_{d}, \text { and } Y_{e}
$$

are the only flavor spurions. They transform under the flavor symmetries as

$$
\begin{array}{c|c|c|c|c|c} 
& U(3)_{Q} & U(3)_{u} & U(3)_{d} & U(3)_{L} & U(3)_{e} \\
\hline Y_{u} & 3 & \overline{3} & 1 & 1 & 1 \\
Y_{d} & 3 & 1 & \overline{3} & 1 & 1 \\
Y_{e} & 1 & 1 & 1 & 3 & \overline{3}
\end{array}
$$

After setting them to their background values, they break the flavor symmetries into sub-groups.

We discuss the quark flavors first. The Yukawa couplings breaks the flavor symmetries down to the Baryon number symmtrey $U(1)_{B}$

$$
U(3)_{Q} \times U(3)_{u} \times U(3)_{d} \rightarrow U(1)_{B}
$$

The two Yukawa matrices $Y_{u}$ and $Y_{d}$ can have 18 real and 18 imaginary parameters. Using the $U(3)_{Q} \times U(3)_{u} \times U(3)_{d}$ rotations (each $\mathrm{U}(3)$ has 3 real transformations and 6 phase rotations), we can remove 9 real and 17 imaginary parameters. We can not remove all 18 imaginary parameters because there is a residual unbroken $U(1)_{B}$ symmetry, which means that there is an $U(1)$ rotation which leaves the quark Yukawas invariant. Hence, in the quark flavor sector, we are left with 9 real parameters and 1 phase. This has the important implication that 3 generations of massive quarks allows for the possibility of CP violation. And, it turns out that Nature does take this opportunity to break CP.

It is often convenient to diagonalize the quark mass matrices and work with the mass eigenstates (denoted as $\hat{u}$ and $\hat{d}$ ). In the Standard Model, this is achieved by

$$
\hat{u}_{L, R}=V_{L, R}^{u} u_{L, R}, \quad \hat{d}_{L, R}=V_{L, R}^{d}=d_{L, R},
$$

where $V_{L, R}^{u, d} \mathrm{~s}$ are $3 \times 3$ unitary matrices, and

$$
\begin{gathered}
\hat{Y}_{u}=\left(\begin{array}{ccc}
\hat{y}_{u} & & \\
& \hat{y}_{c} & \\
& & \hat{y}_{t}
\end{array}\right)=V_{L}^{u} Y_{u} V_{R}^{u^{\dagger}}, \\
\hat{Y}_{d}=\left(\begin{array}{ccc}
\hat{y}_{d} & & \\
& \hat{y}_{s} & \\
& & \hat{y}_{b}
\end{array}\right)=V_{L}^{d} Y_{d} V_{R}^{d \dagger} .
\end{gathered}
$$


The quark masses are $m_{q}=(1 / \sqrt{2}) \hat{y}_{q} v$. These rotations are symmetries of the QCD Lagrangian, and they do not induce changes in the quark-gluon couplings. For the electroweak sector, it has interesting effects. First of all, the so-called neutral current interactions are of the form

$$
u_{L, R}^{\dagger} \bar{\sigma}^{\mu} u_{L, R} A_{\mu}, d_{L, R}^{\dagger} \bar{\sigma}^{\mu} d_{L, R} A_{\mu}
$$

where $A_{\mu}=Z, \gamma$ is a neutral gauge boson. Going to mass eigenstates leaves these interactions diagonal

$$
\left(\hat{u}_{L, R}^{\dagger}\right)_{i} \bar{\sigma}^{\mu}\left(\hat{u}_{L, R}\right)_{i} A_{\mu},\left(\hat{d}_{L, R}^{\dagger}\right)_{i} \bar{\sigma}^{\mu}\left(\hat{d}_{L, R}\right)_{i} A_{\mu}
$$

where we have used index $i=1,2,3$ to label mass eigenstates. This lack of tree level flavor changing neutral current (FCNC) is an important feature of the Standard Model flavor physics. On the other hand, the charge current interactions are

$$
u_{L}^{\dagger} \bar{\sigma}^{\mu} d_{L} W_{\mu}^{+}+\text {h.c. }=\left(\hat{u}_{L}^{\dagger}\right)_{i}\left[V_{L}^{u} V_{L}^{d \dagger}\right]_{i j}\left(\hat{d}_{L}\right)_{j} W_{\mu}^{+}+\text {h.c. }
$$

There is tree level flavor violations in charge current interaction, parameterized by a $3 \times 3$ unitary matrix $V_{L}^{u} V_{L}^{d \dagger} \equiv V_{C K M}$ [20]. The physical flavor parameters are 6 quark masses, 3 mixing angles, and one $\mathrm{CP}$ violating phase. The CKM matrix $V_{C K M}$ contains the mixing angles and the phase. Typically, the CKM matrix can be parameterized as [21]

$$
V_{C K M}=\left(\begin{array}{ccc}
V_{u d} & V_{u s} & V_{u b} \\
V_{c d} & V_{c s} & V_{c b} \\
V_{t d} & V_{t s} & V_{t b}
\end{array}\right)=\left(\begin{array}{ccc}
1-\frac{\lambda^{2}}{2} & \lambda & A \lambda^{3}(\rho-i \eta) \\
-\lambda & 1-\frac{\lambda^{2}}{2} & A \lambda^{2} \\
A \lambda^{3}(1-\rho-i \eta) & -A \lambda^{2} & 1
\end{array}\right)+\mathscr{O}\left(\lambda^{4}\right),
$$

with

$$
\lambda \simeq 0.225, A \simeq 0.82, \rho \simeq 0.14, \eta \simeq 0.35,
$$

where the numerical values are approximately and do not reflect the current precision on them $[12,22]$. We have adopted the so called Wolfenstein parameterization which nicely captured the hierarchical nature of the flavor mixings in a power series of a small parameter $\lambda$. The complex parameter $\rho+i \eta$ measures the size of $\mathrm{CP}$ violation. Available data provides precise measurement of the mixing matrix element, and tests the Standard Model. For example, one way to test the Standard Model flavor structure is to check whether the CKM matrix is indeed unitary. If there is new physics which can induce flavor changing processes, the measured CKM matrix elements, extracted from experiments assuming that there is no addition source of flavor violation, will fail to be unitary [22].

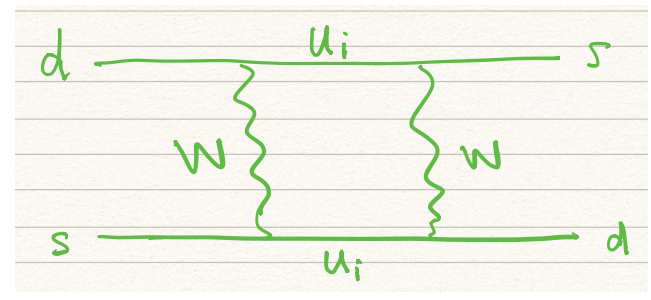

Figure 6: An example of Kaon mixing diagram. 
One of the best measured flavor violation process in the Standard Model is the neutral Kaon mixing. We can estimate

$$
\mathscr{O} \simeq \frac{1}{16 \pi^{2}} \Sigma_{i} \frac{m_{u_{i}}^{2}}{m_{W}^{4}}\left(V_{i d}^{*} V_{i s}\right)^{2}\left(\bar{d}_{L} \gamma_{\mu} s_{L}\right)^{2}
$$

where we have summed over 3 flavors of up type quarks inside the loop, with corresponding CKM mixing parameters as couplings. The factor of $m_{W}^{-4}$ comes from integrating out the W-boson since it is much heavier than the relevant scale $m_{K}$ of this problem. Any other possible contribution without quark masses would be vanishing due to the unitarity of the CKM matrix. Two power of quark masses are needed. One single mass insertion would flip the chirality of the quark line. However, W-boson only couples to left-handed quarks, which forces us to add another quark mass insertion to "flip it back". This operator generate a mixing between the two neutral Kaons $K^{0}$ and $\bar{K}^{0}$, produces two new mass eigenstates $K_{L}$ and $K_{S}$ (named so after the difference in their lifetimes) with

$$
\frac{m_{K_{L}}-m_{K_{S}}}{m_{K}}=\frac{\Delta m_{K}}{m_{K}} \sim 10^{-14},
$$

which has been very well tested by the experimental measurements.

This is a good example for another interesting feature of the flavor violation in the Standard Model. Notice that the mixing operator vanishes in the limit of

$$
m_{u}=m_{c}=m_{t},
$$

due to the unitarity of the CKM matrix. This is the so called GIM mechanism [23]. One way to understand this is that, in this limit, there is an enhanced diagonal flavor symmetry (even though the individual left and right flavor symmetries are broken)

$$
S U(3)_{\mathrm{diag}}=S U(3)_{Q}=S U(3)_{u} .
$$

This is general. Any flavor changing neutral current in the Standard Model is proportional to the difference in quark masses. Due to this effect, even before the discoveries of the top and charm quark, we can already estimate their masses based on the measured flavor violation.

New physics can certainly give rise to new sources of flavor violation. At dimension 6, they can have the form

$$
\frac{1}{M^{2}} q_{i} q_{j} q_{i^{\prime}} q_{j^{\prime}}
$$

where we have suppressed Lorentz structure of the operator. Flavor violation measurements put stringent limits on the sizes of these operators. One of the strongest constraints is from Kaon mixing, which implies

$$
M>10^{7} \mathrm{GeV}
$$

We move on to discuss lepton flavor physics. Within the Standard Model renormalizable interactions (with only Standard Model degrees of freedom), the only spurion is the charge lepton Yukawa matrix $Y_{e}$. It breaks the lepton flavor symmetries as

$$
U(3)_{L} \times U(3)_{e} \rightarrow U(1)_{e} \times U(1)_{\mu} \times U(1)_{\tau}
$$

A counting similar to the quark case tells us there are only 3 real parameters in this case $m_{e}, m_{\mu}$, and $m_{\tau}$, and there is no flavor mixing. However, we know now that this is not complete. Neutrinos have 
masses, and there has to be something more. We have already discussed, depending whether lepton number is a good symmetry or not, there are two ways of adding neutrino masses and mixings. If we preserve lepton number assiciated with each generation of leptons, we could add 3 light right handed neutrinos $\left(n_{i}\right)$ and a new Yukawa coupling $Y_{v}$. In this case, two spurions $Y_{e}$ and $Y_{v}$ break the flavor symmetries

$$
U(3)_{L} \times U(3)_{e} \times U(3)_{n} \rightarrow U(1)_{\text {Lepton number }}
$$

Alternatively, we can write the Weinberg operator with flavor structures

$$
\frac{(H L)_{i}(H L)_{j}}{M}, \text { with } M \sim 10^{12-13} \mathrm{GeV} .
$$

In this case, the Weinberg operator breaks the flavor symmetries

$$
U(3)_{L} \times U(3)_{e} \rightarrow \text { nothing. }
$$

Either way, we can now summarize lepton flavor mixing in the Standard Model charge current couplings by a $3 \times 3$ unitary matrix [24]

$$
V_{P M N S} \equiv V_{L}^{\nu} V_{L}^{e \dagger}=\left(\begin{array}{ccc}
1 & 0 & 0 \\
0 & c_{23} & s_{23} \\
0 & -s_{23} & c_{23}
\end{array}\right)\left(\begin{array}{ccc}
c_{13} & 0 & s_{13} e^{i \delta_{\mathrm{CP}}} \\
0 & 1 & 0 \\
-s_{13} e^{i \delta_{\mathrm{CP}}} & 0 & c_{13}
\end{array}\right)\left(\begin{array}{ccc}
c_{12} & s_{12} & 0 \\
-s_{12} & c_{12} & 0 \\
0 & 0 & 1
\end{array}\right) \text {, }
$$

where $c_{i j}$ and $s_{i j}$ denote $\cos \theta_{i j}$ and $\sin \theta_{i j}$, respectively. We have

$$
\sin ^{2} \theta_{12} \simeq 0.3, \sin ^{2} \theta_{23} \simeq 0.5, \sin ^{2} \theta_{13} \sim 0.02, \delta_{\mathrm{CP}} \sim 230^{\circ} \pm 30^{\circ} .
$$

The numbers are approximate and do not reflect current accuracies. As we can see, there is a major difference between these and the mixings of quark sector. The mixings in the lepton sector are large (in particular for the 12 and 23 mixings), and not hierarchical. There is still no tree level flavor changing neutral current for the leptons. New physics can also give new lepton flavor violation. At dimension 6, they can come in as, for example,

$$
\frac{1}{M^{2}} L_{i} H \sigma_{\mu v} e_{j} B^{\mu v}, \frac{1}{M^{2}} L_{i} \gamma_{\mu} L_{j} L_{i^{\prime}} \gamma_{\mu} L_{j^{\prime}}
$$

Most stringent tests of the lepton flavor violation come from the measurements of processes $\mu \rightarrow e \gamma$ and $\mu \rightarrow e e e$, which roughly set the limit

$$
M>10^{5} \mathrm{GeV}
$$

\section{Beyond the Standard Model}

New physics beyond the Standard Model is a huge field, and it is impossible to cover them in detail. I will focus on giving an overview of the main motivations of thinking about new physics beyond the Standard Model, and offering brief comments on some of the most popular ideas. Due to the lack of experimental evidence, such an overview will necessarily be speculative, and hence subjective. 
It is important to emphasize that the Standard Model is far from a complete theory. Sometimes, it is said that the discovery of the Higgs boson completes the Standard Model. This is true in a very narrow sense. Before the discovery, based on consistency of the theory (recall our discussion about the $W W$ scattering), we know something must be there below $1 \mathrm{TeV}$. The Higgs boson filled that role. With it, in principle, the Standard Model can be a valid effective field theory up to energies way above the weak scale. However, it is not the same as a complete theory. We have no explanation for the basic structure of the Standard Model, such the rather complicated gauge dynamics and the presence of 3 generations. There are 25 free parameters in the Standard Model. After decades of experimental studies, we have learned sizes of almost all of them with different precisions. We will no doubt learn about them better in the future. However, we don't have explanations for the size of anyone of them. We firmly believe the Standard Model can be explained in terms of a more fundamental theory. Figuring out how it works is certainly the main goal of particle physics.

The main part of this section contains a discussion on the so called hierarchy problem associated with the electroweak scale. Before going to this topic, we will very briefly mention other main areas of new physics beyond the Standard Model.

1. Dark Matter. Arguably, the most concrete evidence of the existence of new physics beyond the Standard Model is the dark matter in the universe. This has been supported by a myriad of observational evidence, through the gravitation effect dark matter on the evolution in the early universe, formation of the structure, and dynamics of galaxies and clusters. Hence, strictly speaking, instead of discovering dark matter, the goal is to understand more about what it is. The key step would be to find them in searches which do not rely on their gravitational coupling. It is well established that dark matter can not be a particle within the Standard Model. Beyond that, we know very little about the properties of dark matter. For example, the range of the mass of dark matter particle/object can span more than 80 orders of magnitude, from $10^{-23} \mathrm{eV}$ to over a solar mass. The coupling of the dark matter to SM particles can vary over many decades as well. Therefore, the search for dark matter is literally like shooting in the dark, and it could be a long and difficult process. In particular, it is impossible to do broad model independent searches, and we would need good "stories" to get started. To this end, there are two classes of well established ideas of dark matter, the Weakly Interacting Massive Particle (WIMP) and the Axion [25]. WIMP is based a set of simple ideas. It assumes that the dark matter particle is in thermal equilibrium with the SM particles in the early universe, mediate either through SM interactions or by exotic intermediate particles in more elaborated models. With such a model, we can compute the relic abundance of dark matter today. If we assume that dark matter particles have the masses and couplings strength similar to that of the SM weak scale particles and their interactions, it turns out its abundance is in the right ball park to match the current observation. This coincidence with the SM weak interaction has been a major motivation to consider WIMP as a leading candidate for dark matter. Because it typically has sizable coupling with the SM particles, the WIMP hypothesis can be (and has been) subject to a number of experimental searches, including cosmic ray signals from dark matter annihilation, direct detection of dark matter nucleus scattering in underground labs, and direct production of dark matter particles at high energy colliders. I consider the main strength of the WIMP scenario lies in 
the simplicity of the basic assumption, and the fact that it's insensitive to earlier evolution of the universe. In addition, if WIMP dark matter is discovered, we can measure its properties in the lab. From that, we can compute its relic abundance. It has the potential of replicate the success of the Big Bang Nuclear Synthesis, which is a corner stone of modern cosmology. The Axion scenario is motivated by another nagging problem of the SM, the strong $\mathrm{CP}$ problem. It is an interesting candidate of dark matter, and it gives a completely different set of signatures. The main approaches of the axion searches rely on its coupling to photons. If axion is the solution of the strong CP problem, there is a almost model independent lower bound on the axion-photon coupling due to its mixing with $\pi^{0}$. Of course, we could also consider an axion-like particle, which may not have a direct connection with the strong CP problem. There have been many variations of axion models. However, most of them fall in these two broad categories. More recently, there are new interest in more exotic scenarios such as ultra-light dark matter (all the way to the lower limit) or macroscopic objects such as primordial blackholes. These are all interesting venues to pursue. On the other hand, the stories in these alternative models are not as well developed as the WIMP and axion.

2. Matter anti-matter asymmetry in the universe. It is an obvious fact that there is more matter than anti-matter in our universe. One could attribute this to some initial condition. However, the presence of an attractor, such as a period of inflation, in the early universe certainly makes this less plausible. Another, and more appealing, possibility is to generate such an asymmetry in the later evolution of the universe after the hot big bang (reheating). This is the so called Baryogenesis. With some mild assumptions, successful baryogenesis requires a set of special conditions, both on the fundamental parameters ( $\mathrm{C}$ and $\mathrm{CP}$ violation, B or $\mathrm{L}$ violation), and on the evolution history (out of thermal equilibrium). Remarkably, the SM can not satisfy these conditions to give rise to the observed asymmetry. This is another major open question facing particle physics. One of the leading candidates for baryogenesis is the Leptogenesis[19]. It fits well with the simple see-saw model of neutrino mass generation. At the same time, since the right-handed neutrino in the simplest scenario is very heavy $\left(\sim 10^{13}\right.$ $\mathrm{GeV}$ ), testing it directly is difficult. There can be indirect evidence from the measurement of the properties of the neutrinos, such as $\mathrm{CP}$ violation and lepton number violation. Another leading candidate is called Electroweak Baryogenesis [26]. In this scenario, a first order electroweak phase transition gives rise to the out of equilibrium process. In addition, new sources of $\mathrm{CP}$ violation and $\mathrm{B}$ or $\mathrm{L}$ violation need to be introduced. All of these require the addition of new physics. Moreover, the new physics particles need to be close the weak scale and have a sizable coupling with the SM electroweak sector. This makes the electroweak baryogenesis scenario the most testable one. For the same reason, it is also the most constrained. Some of the once popular models, such as its realization in minimal supersymmetric models with light stops, have been disfavored by experimental constraints already. Nevertheless, in my view, it is still a compelling and exciting scenario. Its close connection to the electroweak sector means that it could have a connection to the solution of the hierarchy problem (to be discussed later in this section).

3. Flavor and CP. The Standard Model has an intriguing flavor structure. There are three families of quarks and leptons. They have masses which differ by as much as 10 orders 
of magnitudes. They have mixings. In the quark sector, the mixings are hierarchical. On the other hand, in the lepton sector, the mixings are large. The presence of CP violation in the quark sector has been well established, while there is already some evidence of the presence of a large $\mathrm{CP}$ violation in the lepton sector. Overall, the flavor structure in the SM looks like a big mess, and we don't know where it comes from. In the SM, the flavor structure is parameterized through a set of Yukawa couplings. It is worth emphasizing these are coefficients of marginal operators. This means we do not have an expectation of the scale associated with the generation of these operators. Therefore, it can be difficult to test these models. We do have a lot of ideas, and there are a lot of models. For example, the SM fermions can have interactions with some additional scalars (called flavons) and heavy exotic fermions. The Yukawa couplings can be generated once the heavy states are integrated out and flavons acquire vacuum expectation values. A hierarchical structure is possible if the coupling depends on different powers of the flavon VEV, controlled either by some flavor symmetries or some other dynamics [27]. This kind of scenarios have a lot of variations, and can be implemented in many scenarios, such as in Grand Unified Theories (GUT). However, it only gives the Yukawa couplings in terms of the ratio between the flavon VEV and the masses of the heavy fermions, i.e., it does not prefer a specific scale. This is a manifestation of the fact that marginal operators do not directly point to a scale. If this is the way flavor structure is generated in the SM, we can test it only if the flavor scale happens to be low. We need to be lucky. There is one class of models for which the flavor scale has to be close to the weak scale. This is the composite Higgs model, which we will discuss in some detail later. In this set of models, since the Higgs is composite, at least some of the heavy new physics states involved in the generation of the Yukawa coupling would be close to the weak scale. They can be either some composite resonances or some additional elementary particle. Because of this, the origin of flavor structure in the composite Higgs models is more testable, and there has already been stringent limits. There is yet another source of $\mathrm{CP}$ violation in the SM. This comes from a misalgnment between the QCD vacuum angle and the quark mass matrices. To be consistent with the experiments, mostly from the neutron electric dipole moment measurement, the size of the $\mathrm{CP}$ angle from this source needs to be less than about $10^{-9}$. Without a symmetry or dynamical mechanism, this seems to an unusually small number. This is the so called strong $\mathrm{CP}$ problem. The most popular solution for this problem is to promote the $\mathrm{CP}$ angle into a dynamical field, whose vacuum expectation value can relax the $\mathrm{CP}$ angle down to zero. There is a striking prediction of this scenario, namely there would be a light Goldstone boson, called axion. We have mentioned this possibility in our discussion on dark matter candidates.

4. Dark Energy. Most of the energy in the universe, about $70 \%$, is of a form which is very different from regular matter. It is called dark energy. Observation of the dark energy so far is consistent with it being a constant vacuum energy, a.k.a., the cosmological constant. It is a component of energy which is allowed by Einstein's equation, which can be a free parameter. To match observation, we need the cosmological constant to be roughly $\Lambda^{4} \sim\left(m_{W}^{2} / M_{\text {Planck }}\right)^{4}$. However, problem arise as soon as we try to predict this number from a fundamental theory. In fact, as we will discuss in more detail later in the context of the electroweak hierarchy 
problem, any fundamental theory with high mass thresholds $\sim M$ will give large contribution to the vacuum energy $\propto M^{4}$. Suppose the fundamental physics which determines the cosmological constant is at the Planck scale, the naive prediction would be $10^{120}$ times larger than the observed value. It certainly has been called, with some justification, the most spectacular failure of the prediction of a theory. One might think we can be smarter and find some clever mechanism to generate such a small number. The apparent numerical coincidence between the cosmological constant, the weak scale, and the Planck scale shown above seems to already give some clue what a successful mechanism could be. However, it turns out to be very difficult. Many such mechanisms do reduce the magnitude of cosmological constant by a lot, however, there still seems to be at least a factor of $10^{60}$ no matter what we do. Therefore, we are left with searching for really outside-of-the-box type of ideas. An intriguing argument has been provided by Weinberg [28]. It starts with WIMP dark matter with interaction cross section with the SM of the order $\sim \alpha_{\mathrm{D}}^{2} / m_{\mathrm{DM}}^{2}$, where $\alpha_{\mathrm{D}}$ is a typical weak coupling. A density perturbation of $\delta \rho / \rho \sim 10^{-5}$ is generated by inflation, which will start to grow linearly with the scale factor after matter radiation equality. For the structure to form, at least, the vacuum energy can not dominate over dark matter energy density at the time when the desity perturbation grew to be $\delta \rho / \rho \sim 1$. Putting this together, one could get a upper limit on the cosmological constant $\Lambda^{4}<\left(m_{\mathrm{DM}}^{2} / M_{\text {Planck }}\right)^{4}$. This upper limit is close to the observed value if $m_{\mathrm{DM}} \sim v$ which is typical in WIMP models. Obviously, there are several very big assumptions, and a lot of lingering questions. However, the fact that this is only estimate of the cosmological constant which even get remotely close to the right answer compels us to take it seriously. A nautral interpretation of this is in the context of landscape and anthropic principle. These words tend to generate a lot of negative feeling, a lot of which is not fully justified. I don't think we can dismiss this as a possibility. This would then essentially put the determination of the cosmological constant, and the effort of predicting the size of the oribits of the planets in the solar system on the same footing. It's certainly possible that we are indeed missing some crucial piece of physics. For example, since we have only observed the effect of cosmological constant from its gravitational effect at large scales. Perhaps it is gravity on these scales which needs to be modified. Or, perhaps we need to understand the De Sitter space better. Attempts along these lines have all been made, with no success yet. However, until we have definitive proof that cosmological constant is due to selection on the landscape, I think our working assumption should always be we are missing something big in dynamics. It would be more interesting if this is indeed the case, since we will be learning something deep.

\section{1 $m_{W} / M_{\text {Planck }} \simeq 10^{-16}$, the hierarchy problem}

Perhaps the most striking feature of the Standard Model electroweak sector is the presence of the weak scale $\sim 100 \mathrm{GeV}$. The Standard Model contains a very simple picture of the electroweak symmetry breaking, which is implemented by an elementary scalar field with the following potential

$$
V(H)=-\frac{\mu^{2}}{2} H^{\dagger} H+\frac{\lambda}{4}|H|^{4} .
$$




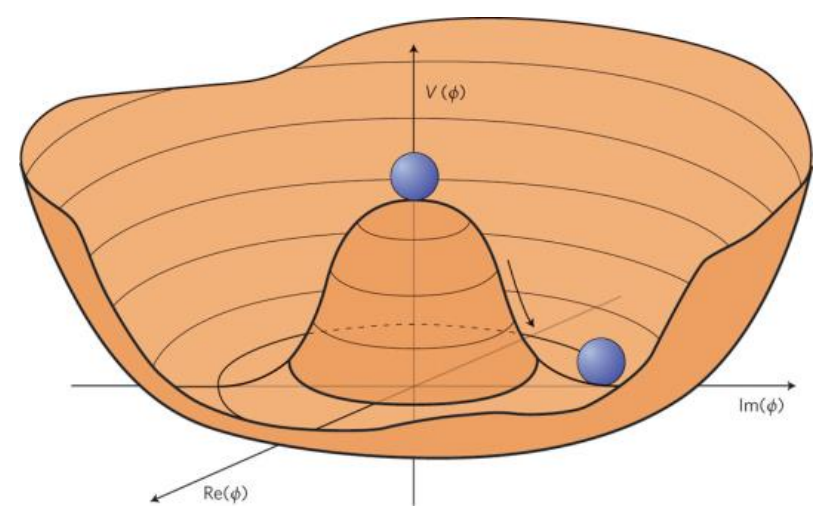

Figure 7: The Mexican Hat potential.

The Higgs field rolls down this so called Mexican Hat potential, acquiring a vacuum expectation value and triggering electroweak symmetry breaking. This is probably the simplest way through which electroweak symmetry breaking can happen. The discovery of the Higgs boson and subsequent measurements of its properties are consistent with this picture. Within this model, we can already infer from experimental measurements the size of the parameters in this Higgs potential to an accuracy of better than a percent.

However, this simplicity is misleading. We would like to explain the electroweak scale in terms of a more fundamental theory. The first question one would like to answer in this endeavor is what the scale of this more fundamental theory is. Strickly speaking, we have no idea. One first guess could be that the underlying fundamental theory lives at the Planck Scale $\sim 10^{18} \mathrm{GeV}$, which is the only known scale above the weak scale. However, an obvious question is how to generate the 16 orders of magnitude difference in the mass scale, $m_{W} / M_{\text {Planck }} \sim 10^{-16}$.

It is often said in the literature that the problem is due to the fact the quantum corrections to the Higgs mass is quadratically divergent. In particular, due to its large coupling to the top quark, the divergence from the top quark loop is especially worrisome. This, at most, is a very hand-wavy way of stating the problem, which can be quite misleading. Indeed, it leads to a wide spread impression that the problem is due to renormalization (and the corresponding regularization procedure). However, this is not accurate. The real question only arises when we attempt to write down a model from which the mass parameter ( $\mu^{2}$ in the Higgs potential) is calculable. This is certainly a requirement for any UV theory which can offer an explanation for the presence of the weak scale. It is meaningless to talk about the so called fine-tuning in the absence of such a UV completion.

To understand the kind of difficulty we might encounter, we consider the following toy model of a scalar $\phi$ interacting with some heavy fermions,

$$
\mathscr{L} \supset M_{\Psi}\left(\bar{\Psi}_{1} \Psi_{1}+\bar{\Psi}_{2} \Psi_{2}\right)+y \phi \bar{\Psi}_{1} \Psi_{2}+\text { h.c. },
$$

where we can assume $y \sim \mathscr{O}(1)$. Following the standard procedure, for example $a$. la. Coleman- 
Weinberg, to integrate out the heavy fermions generates a potential for the scalar of the form

$$
V^{\Psi}(\phi) \simeq \frac{-1}{16 \pi^{2}}\left(a M_{\Psi}^{4}+b M_{\Psi}^{2} y^{2} \phi^{2}+c y^{4} \phi^{4}\right) \times\left(\log \frac{M_{\Psi}^{2}}{\mu^{2}}-\ldots\right) .
$$

$a, b$ and $c$ are computable in our specific toy model. At the same time, they are in general $\mathscr{O}(1)$ numbers depending on the number of fermions, etc. Hence, a generic expectation is that integrating out heavy particles in the underlying theory will generate large corrections to the scalar mass, proportional to the square of the masses of the heavy particles. Of course, this is the usual statement, in particular in Wilsonian effective field theory, that scalar mass operator is quadratically sensitive the UV physics. However, understanding it as an effect of integrating out heavy particles, which are generically present in any UV model, makes it obvious this is not a question of regularization scheme or renormalization of some divergence. It is a general expectation of the prediction of a UV theory.

As a side remark, we notice that the first term in Eq. 3.3 is a contribution to the vacuum energy proportional to $M_{\Psi}^{4}$. Coupling to gravity, this becomes a large contribution to the cosmological constant. This is an example of the sensitivity of the cosmological constant to the high energy thresholds in fundamental theory. It underlies the difficulty in generating the right size for the cosmological constant. The logic is very similar. However, numerically, it is much worse than the difficulties in predicting the Higgs mass.

Integrating out fermions gives large negative contributions. However, we can also imagine that the UV theory also contain heavy scalars. Again, we can consider a toy model

$$
\mathscr{L} \supset \frac{M_{\Phi}^{2}}{2} \Phi^{2}+\frac{\kappa}{2} \phi^{2} \Phi^{2}
$$

where $\Phi$ is a heavy real scalar. We can integrate out $\Phi$ and obtain

$$
V^{\Phi}(\phi) \simeq \frac{1}{16 \pi^{2}}\left(a^{\prime} M_{\Phi}^{4}+b^{\prime} \kappa^{2} M_{\Phi}^{2} \phi^{2}+c^{\prime} \kappa^{4} \phi^{4}\right)\left(\log \frac{M_{\Phi}^{2}}{\mu^{2}}+\ldots\right),
$$

and $a^{\prime}, b^{\prime}$ and $c^{\prime}$ are again $\mathscr{O}(1)$ numbers.

Obviously, in a general UV model, it is possible to choose masses such as $M_{\Psi}$ and $M_{\Phi}$, and couplings such as $y$ and $\kappa$ (and possibly adding a "bare" mass term for $\phi$ ), to obtain an low energy effecive potential

$$
V_{\mathrm{eff}}(\phi)=\frac{1}{2} \mu^{2} \phi^{2}+\frac{\lambda}{4} \phi^{4}
$$

with $\mu^{2} \ll M_{\Phi}, M_{\Psi}$. However, large cancellation among various terms with a precision on the order of

$$
\text { cancellation }: \sim \mathscr{O}\left(16 \pi^{2} \frac{\mu^{2}}{M_{\Psi, \Phi}^{2}}\right)
$$

is necessary. This is sometimes called fine-tuning, although no external tunable knob is implied. For example, going back to our simple guess that $M_{\Phi, \Psi} \sim M_{\text {Planck }}$, we would expect a cancellation to a level about $10^{-32}$. As usual, without any specific reasons (such as symmetries), we do not expect such cancellations. 


\begin{tabular}{cc|ccc} 
& spin & & & spin \\
gluon, g & 1 & gluino & $\tilde{g}$ & $1 / 2$ \\
$\mathrm{~W}^{ \pm}, \mathrm{Z}$ & 1 & gaugino & $\tilde{W}^{ \pm}, \tilde{Z}$ & $1 / 2$ \\
quark & $1 / 2$ & squark & $\tilde{q}$ & 0 \\
$\ldots$. & & $\ldots$ & \\
Standard Model particles & \multicolumn{2}{|c}{ superpartners }
\end{tabular}

Figure 8: Actors in the SUSY story.

There are two main classes of ideas of avoiding such an artificial large cancellation. The first is to have some symmetry which can gaurantee such a cancellation. A prime example of this is Supersymmetry (SUSY) $[29,30]$. There is no place here to give a detailed introduction. We will just focus on its broad feastures and make a few comments. SUSY is symmetry between fermionic and bosonic states in a theory which share the same quantum numbers otherwise. Application in particle typically focuses on the simplest case, the $N=1$ SUSY. Applying it to the Standard Model, this leads to the presence of a supersymmetric partner (superpartner) for each of the Standard Model particle. An interesting property of SUSY is that the quadrative sensitivity to the heavy (and supersymmetric) mass thresholds precisely cancel to all orders of perturbation expansion. Known as the non-renormalization theorem [31], it says the dependence on the high mass thresholds is at most logarithmic. Explaining the proof of the theorem is beyond our scope here, we can only offer a (very) slick argument. It is well known that fermion mass only has logarithmic dependence on the heavy threshold. For example, in a theory with a Yukawa interaction

$$
\mathscr{L} \supset m_{\psi} \bar{\psi} \psi+y \Phi \bar{\psi} \psi+\text { h.c. }+\frac{1}{2} M_{\Phi}^{2}|\Phi|^{2},
$$

integrating out the heavy scalar $\Phi$ only generates a correction to the fermion mass

$$
\delta m_{\psi} \propto \frac{|y|^{2}}{16 \pi^{2}} m_{\psi} \log \left(\frac{m_{\Phi}^{2}}{m_{\psi}^{2}}\right),
$$

which is logarithmic in the heavy scalar mass $m_{\Phi}$, instead of the naive expectation from dimensional analysis $\delta m_{\psi} \propto m_{\Phi}$. This is due to the fact that fermion mass breaks a chiral symmetry under which the fermion transforms as

$$
\psi \rightarrow e^{i \gamma^{5} \alpha} \psi
$$

In this theory, the fermion mass $m_{\psi}$ is the only spurion of the chiral symmetry, and any correction to the fermion mass has to be proportional to $m_{\psi}$. Hence, any dependence on the high mass scale $M_{\Phi}$ is at most logarithmic. Now, supersymmetry is a symmetry between fermions and scalars. 
Therefore, combining with the chiral symmetries which protect fermion masses, supersymmetry implies that the scalar masses also at most have logarithmic sensitivity to the high mass thresholds.

Generically, SUSY predicts the weak scale as

$$
\frac{m_{W, Z}^{2}}{\Lambda_{\mathrm{SUSY}}^{2}} \sim e^{-\frac{16 \pi^{2}}{y_{\mathrm{top}}^{2}}} .
$$

$\Lambda_{\text {SUSY }}$ is the "input" scale at which the parameters of the SUSY theory is given. This can be the Planck scale, or the (smaller) string scale, or the scale of grand unification (GUT) $\Lambda_{\mathrm{GUT}} \sim 10^{16}$ $\mathrm{GeV}$. The expoentially large scale separation is possible due to a slow logarithmic (guaranteed by SUSY) "running" of the parameters. It is a story close to the dimension transmutation in QCD. The appearance of the top Yukawa coupling, $y_{\text {top }} \sim 1$, in the exponential is meant to indicate it as the dominant driver of the evolution. In reality, there is more to this story. Theloop with top quark (and its superpartner, $\tilde{t}$, called stop) will have a color factor of 3 . At the same time, there are also other (not totally negilible) contributions, such as from gauge interactions, which can have a different sign. At the same time, it is not a stretch to believe that a scale separation of about 30 orders of magnitude is not hard to generate in SUSY this way.

However, this is far from the full story. Perhaps the largest piece we have not included is the fact that SUSY must be a broken symmetry, since we haven't seen the superpartners predicted by SUSY with the same masses as the Standard Model particles. Therefore, there is another scale in the story the mass gap between between the Standard Model particles and their superpartners. There are many such parameters. However, to continue our qualitative discussion, we won't go into too much details, and we will just denote them collectively as $m_{\text {soft }}$ unless specified otherwise. The subscript "soft" refers to a somewhat more restricted set of SUSY breaking parameters which are deemed to be soft in that they don't reintroduce the power law sensitivity to high energy scales. It is worth noting that, typically, one does get the soft breaking in a fairly straightforward way. It takes some extra-effort to generate "hard" breaking terms.

This set of SUSY breaking parameters $m_{\text {soft }}$ plays a very important role in the dynamics of electroweak symmetry breaking. In particular, we have (schematically)

$$
m_{W, Z}^{2}=m_{\text {soft }}^{2}\left[1-\frac{3}{8 \pi^{2}} \log \left(\frac{\Lambda_{\text {SUSY }}^{2}}{m_{W, Z}^{2}}+\ldots\right)\right] .
$$

The $m_{\text {soft }}^{2}$ in front is setting the scale of the Higgs potential. This is expected since $m_{\text {soft }}$ is the mass scale of the new particles, analogous to the heavy fermion and boson in our toy model. The only difference here is that the Higgs mass itself receive tree level contribution from SUSY breaking mass parameters as well. We see that if we would like to completely avoid the fine adjustment of the mass parameters in the UV theory to produce the electroweak scale, we would like the size of SUSY breaking parameters not to be too much heavier than the weak scale itself. This is the expectation that avoiding fine-tuning in SUSY needs the masses of the superpartners to be close to the weak scale. And, the fact that LHC have not found any superpartner yet around this scale is causing some amount of strain in the naturalness of the SUSY models.

In more detail, the constraints on different SUSY breaking parameters are quite different. The one superpartner whose mass has become the standard measure of fine-tuning is the superpartner 
of the top quark, called stop $\tilde{t}$. We should explain its role in the electroweak symmetry breaking in SUSY, since such a role is similar for other so called top partners in other non-SUSY models as well. As we have remarked earlier, a UV completion should make the mass parameters in the Higgs potential calculable. Or at least, if there is some UV physics being "swept under the rug " (as it is usually the case), one should aim at reducing the sensitivity of weak scale to those unspecified details as much as possible. In SUSY limit, this is achieved through the fact that the theory has only at most logarithmic sensitivity to the UV physics. From bottom up point of view, this means the corrections to the Higgs potential from the Standard Model field must be "regulated" by the contribution of the new physics. In the Standard Model, the largest contribution to the Higgs potential comes from the top quark. Hence, new physics would need to have a top-partner which could play the role of regulating this contribution. In SUSY, this role is filled by the top squark. The calculable contribution to the Higgs potential from the top sector is then

$$
\delta V^{\text {top-stop }} \simeq-\frac{3}{8 \pi^{2}} m_{\tilde{t}}^{2} H^{\dagger} H \times \log \left(\frac{\Lambda_{\text {SUSY }}}{m_{\tilde{t}}}\right)+\ldots
$$

To avoid this generates too large a mass term in the Higgs potential, we would like to have this less than about $\left(10^{2} \mathrm{GeV}\right)^{2} H^{\dagger} H$. At this moment, the search for stop has put a limit to about $m_{\tilde{t}}>1$ $\mathrm{TeV}$. We will leave it as an exercise to decide whether this is too much for the naturalness of SUSY.

Much of the SUSY phenomenology has been focused on the case of the so called Minimal Supersymmetric Standard Model (MSSM). It takes the Standard Model matter content and "supersymmtrizes" it by putting particles into super-multiplets. It is a appealing model since it is minimal, and historically, it has played an very important role in SUSY phenomenology. In this particular model, however, the fine-tuning is somewhat more severe. This comes from the requirement to predict the correct mass of the Higgs boson (not just the size of the mass parameter in the Higgs potential). To this end, we recall from a general Mexican hat potential, after expending around the true vacuum, the mass of the Higgs boson is

$$
m_{h}^{2} \simeq \lambda v^{2}
$$

In practice, the vacuum expectation value is fixed by the weak gauge boson mass as $v=246 \mathrm{GeV}$. Hence, in order to calculate the Higgs boson mass, we still need the quartic coupling $\lambda$. In SUSY, at tree level, this quartic coupling is related to the electro-weak gauge couplings, $\lambda \propto g^{2}+g^{\prime 2}$. This leads to a striking prediction that, at tree level,

$$
m_{h}^{2}<m_{Z}^{2}
$$

Unfortunately, $m_{h} \simeq 125 \mathrm{GeV}$. On the other hand, there are large one-loop contribution to the quartic coupling, typically dominated by the stop loops due to the large top Yukawa coupling [32]

$$
\delta \lambda \sim \frac{3}{4 \pi^{2}} y_{t}^{2} \log \left[\frac{m_{\tilde{t}}^{2}}{m_{t}^{2}}\right] .
$$

Unfortunately again, the "usual" size of this correction can only lift the Higgs mass up to about 110 $\mathrm{GeV}$. One way to go up to $125 \mathrm{GeV}$ is to cranking up the stop mass. However, it is not so easy as it is logarithmic. In the simplest case, this can require stop mass to be around 10s (even 100) TeV, 
certainly too heavy for the SUSY to be completely natural. However, one can try to be "smart". The dependence on stop mass is indeed not so simple, and there are several other parameters at out disposal. We can still get stop to be around $1 \mathrm{TeV}$ and Higgs mass to be around $125 \mathrm{GeV}$. One can certainly wonder whether such smartness is another fine-tuning. Further pondering on this point is again left as an exercise.

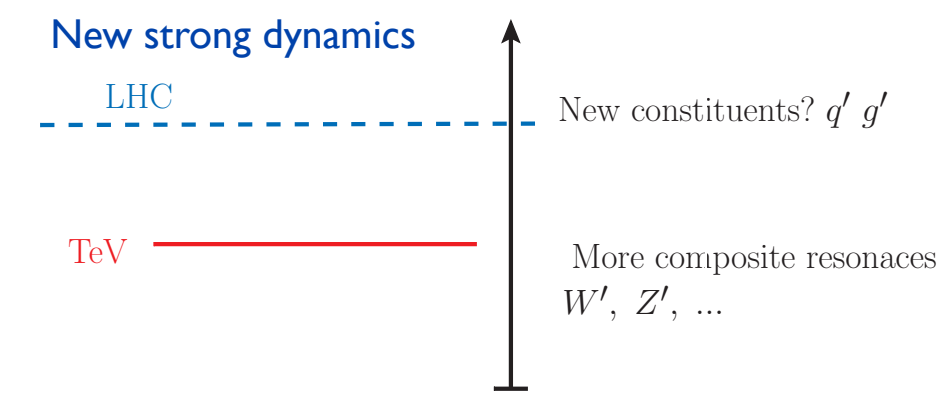

$100 \mathrm{GeV}$

W, Z, Higgs

Figure 9: Scales and resonances in a composite Higgs model.

Having discussed the electroweak symmetry breaking in SUSY, we now move on to a second main class of models, the Composite Higgs [33]. To motivate Composite Higgs, we come back to the low energy physics of the QCD. In this theory, there are low mass scalars, i.e., the mesons. We don't worry too much their naturalness. The reason is that we know they are not elementary. In particular, we know that the QCD has a rather low confinement scale $\Lambda_{\mathrm{QCD}} \sim \mathrm{GeV}$, above which there is no scalar anymore. The lightness of the mesons can be further understood since they are pseudo-Nambu-Goldstone bosons. They are Goldstones from the chiral symmetry breaking, and their masses are calculable from additional small explicit breaking. In addition, we don't worry about the appearance of the low confinement scale $\Lambda_{\mathrm{QCD}} \ll M_{\text {Planck }}$, since it is generated by the slow evolution of the QCD gauge coupling. The idea of Composite Higgs is based on copying the success of the theory of the QCD pions.

The general structure of a Composite Higgs model starts from a global symmetry breaking $G \rightarrow H$ controlled by dynamics of some strong sector. We will weakly gauge some of the global symmetries to accommodate the Standard Model gauge interactions. The coset model has $G / H$ worth of Nambu-Goldstones. Some of them have the correct Standard Model gauge quantum numbers to be the Higgs doublet. Elementary fermions with the same quantum number as the Standard Model ones are introduced. They can mix with the composite fermionic resonances. The resulting light eigenstates, the Standard Model fermions in this model, can be partially composite. Both the fermion couplings and the Standard Model gauge interactions explicitly breaks the global symmetry, generating a potential for the pseudo-Goldstones. This would be the Higgs potential. Again, similar to the SUSY model, there are top partners (fermionic in this case), and they played an important role generating the Higgs potential

$$
V(H) \simeq \frac{3 y_{t}^{2}}{16 \pi^{2}}\left(c_{2} m_{T}^{2} H^{\dagger} H+c_{4} g_{*}^{2}|H|^{4}+\ldots\right),
$$




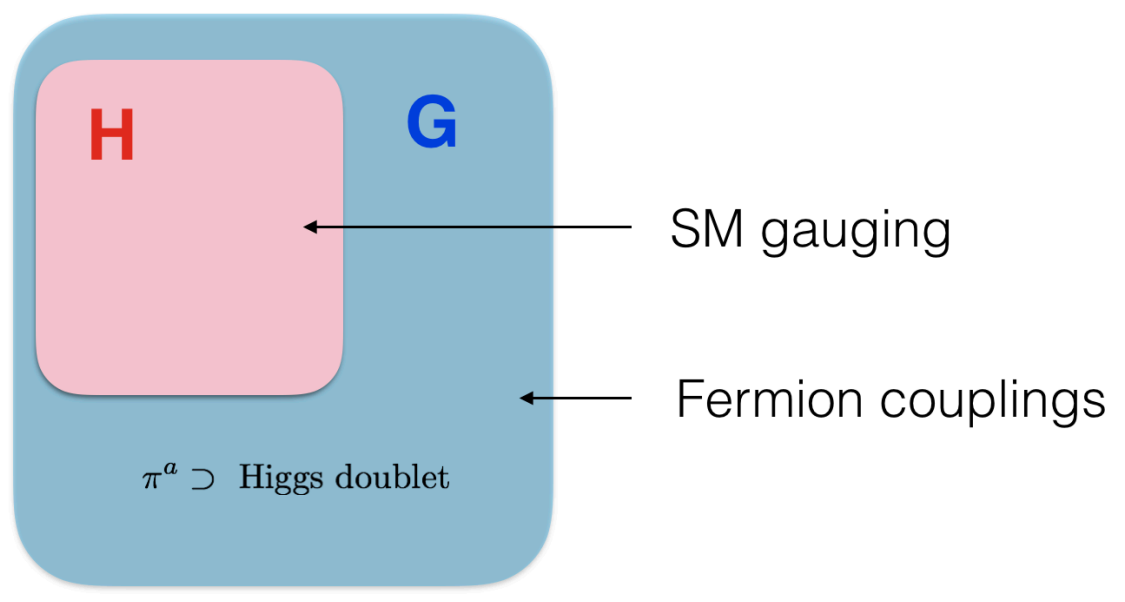

Figure 10: General structure of a composite Higgs model.

where $c_{i}$ s are expected to be $\mathscr{O}(1)$ numbers. $g_{*}$ parameterizes the large coupling from the strong sector. We see that, similar to the case of the SUSY, the contribution from the top partner plays an important role in setting the scale of the Higgs potential. The current limit on the mass of fermionic top partners is also around $1 \mathrm{TeV}$, we are again in some gray area about whether this is already finely tuned. A way to sharpen this tension is through that fact the explicit breaking in a coset model will in general give a potential in as a function of $h / f$, where $h$ is the neutral component of $H$ and $f$ is the analog of the pion decay constant in chiral perturbation theory of QCD. In this case, we generically expect the minimal of the potential would be $v=\langle h\rangle \sim f$. However, typically, a non-linear sigma model

$$
\mathscr{L} \supset \operatorname{tr}(\partial \Sigma \partial \Sigma)+\ldots \text { with } \Sigma=e^{i H / f}
$$

will generate Higgs wave function renormalizations after electroweak symmetry breaking, and will shift the Higgs coupling (for example its coupling to Standard Model gauge bosons) at the level of $\delta \sim v^{2} / f^{2}$. Current limits on such deviations already restrict $f>700 \mathrm{GeV}$. This has already put some strain on the fine tuning. The pressure will only increase with the increase of the precision of the Higgs coupling measurements.

The Composite Higgs model also faces some embarrassment on predicting the mass of the Higgs boson, from an opposite direction. We expect the quartic coupling to be large $\propto g_{*}$, as it is controlled by strong dynamics. As a result, the Higgs mass would be generically too heavy. 
Therefore, none of the two dominant paradigms for natural electroweak symmetry breaking is completely comfortable. Primarily, this is due to the fact that none of the predicted new physics particles have been found yet. In addition, both of these seem to have some trouble in giving the right Higgs boson mass. Such a assessment is certainly way too simplistic. The conclusion can be avoided in carefully chosen part of the parameter space, and of course, it can be (and has been) circumvented in more sophisticated models.

However, it does make us more aware of the possibility that these once considered very well motivated models (and still well motived for many reasons) could be wrong instead. It is definitely not time yet to give up on the experimental searches for these models. At the same time, it is more urgent to think about additional possibilities of solving the naturalness question.

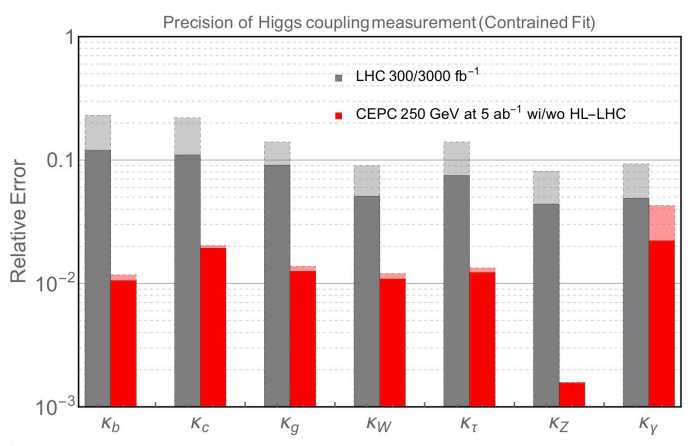

Figure 11: Prospect of Higgs coupling measurement at the LHC, and future lepton collider using the Circular Electron Positron Collider (CEPC) as an example.

It is time like this that experimental guidance can be crucial. At the same time, it can be confusing about where to look for new hints without some guiding principle. Fortunately, for the electroweak symmetry breaking, there is a natural place to begin with. That's the measurement of the properties of the Higgs boson. It is the right place since anything addressing the problem of electroweak scale would have to talk to the Higgs potential, and hence leave imprints on the Higgs couplings.

Currently, the precision on the Higgs coupling measurement is around $5-10 \%$, depending on the coupling. We can translate this into an estimate of the new physics scale. A generic deviation of the Higgs coupling, after integrating out some new physics which couples to the Higgs boson, is expected to be

$$
\delta \simeq c \frac{v^{2}}{M_{\mathrm{NP}}^{2}},
$$

where $M_{\mathrm{NP}}$ is the mass of the new physics states. $c$ is a coupling, which is expected to be at least not too much larger than $\mathscr{O}(1)$. Hence, currently, the LHC is roughly probing the range of $M_{\mathrm{NP}} \sim 700-800 \mathrm{GeV}$. In the longer term, the high luminosity upgrade of the LHC will push this limit to be $4-5 \%$, this is probing the new physics scale to be above $1 \mathrm{TeV}$. Further down the road, there have been proposals of new electron-positron colliders. Both circular (FCC-ee and CEPC) and linear (ILC and CLIC) options have been considered. We will not get into the details of the pros and cons of different options here. Precision measurement of the Higgs coupling is the number one physics case for such colliders. We see that they can push the Higgs coupling measurement to the sub percent level, with reaches the new physics scale of $5-6 \mathrm{TeVs}$. 

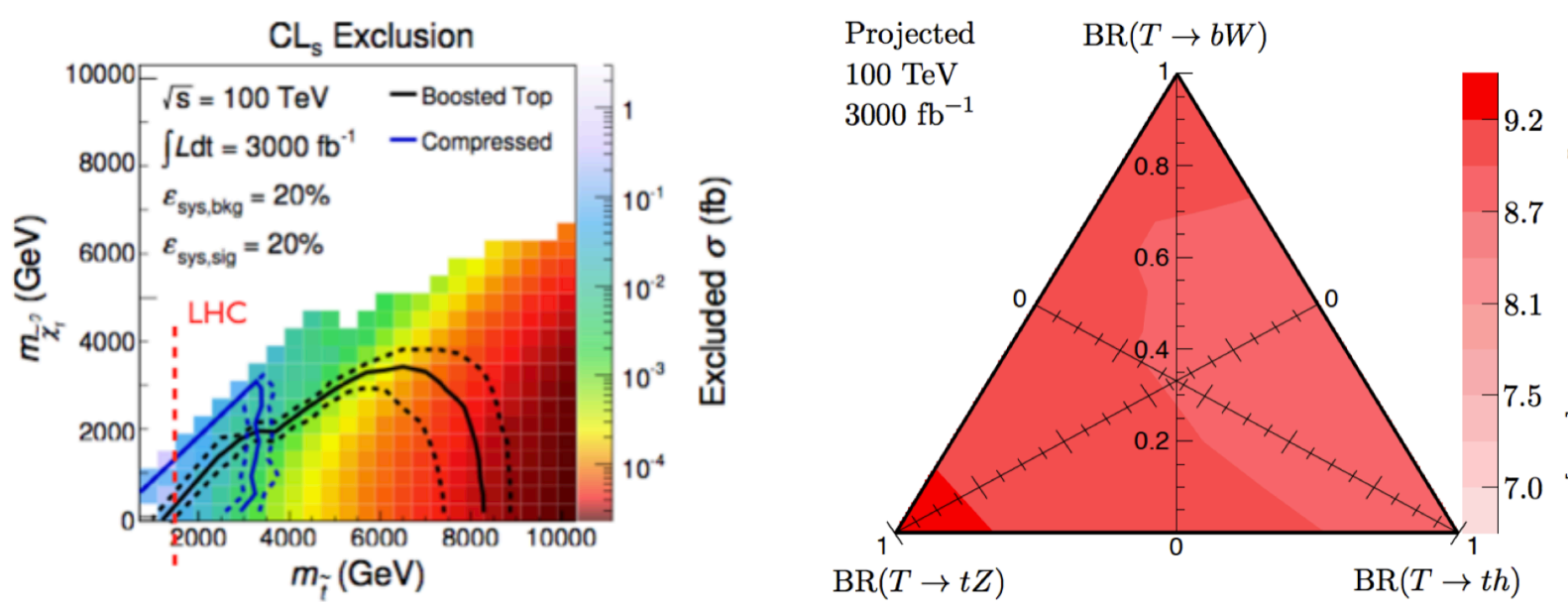

Figure 12: Search of the top partners, at the future proton colliders.

In the mean time, the search for top partners will continue to be the central focus of the naturalness problem. The current limit is about $1 \mathrm{TeV}$. The upcoming runs of the LHC will bring about another $50 \%$ increase in the reach. In the mean time, there have been proposals of more powerful future hadron colliders, such as the HE-LHC with $E_{\mathrm{CM}}=27 \mathrm{TeV}$, and FCC-hh/SPPC. with $E_{\mathrm{CM}}=100 \mathrm{TeV}$. Since the reach of a hadron collider scales approximate linearly with $E_{\mathrm{CM}}$, and the fine tuning scales with $M_{\mathrm{NP}}^{-2}$, we see that these high energy colliders can provide much more stringent tests of the idea of naturalness.

Finally, we offer a couple more comments on naturalness.

1. We have seen that in both SUSY and composite Higgs, there would be tension when the new physics particle is heavier than $\mathrm{TeV}$. It is a generic expectation for new physics models for electroweak symmetry breaking. As we have stressed, the electroweak scale and the Higgs mass are not calculable within the Standard Model. New physics to address this question needs to make it calculable. In all known examples, this is done through regulating the Standard Model contribution with the new physics contribution. Hence, the new physics scale sets the size of this regulation effect, and the Standard Model Higgs potential is quadratically sensitive to it.

2. In both SUSY and composite Higgs models, we have in mind some theory well defined in deep UV potentially up to Planck scale (supersymmetric completion of the Standard Model, or a theory of techni-quark and gluon). This is based on the picture that a high quantum gravity scale is the scale of fundamental physics (and perhaps the ultimate limit of effective field theory). However, it is also possible to consider scenarios in which the fundamental scale, $M_{\text {quantum gravity }}$ is low and not very much beyond $\mathrm{TeV}$. This can be realized in the so called large extra-dimension scenario (on the order of sub-milimeters) [34]. In this case, the weak scale is natural because it is not so different from the quantum gravity scale to begin with. This scenario offers a range of interesting new signals, including modification of gravity at a length scale which can be probed at table top experiments, and direct collider signals from graviton radiations into the extra-dimension(s). Even the physics of quantum 
gravity would not be that far above from our accessible energies. However, in this case, the picture is not quite complete. We still have to explain the weakness of the gravity. This is the same hierarchy problem, just viewing it from a different angle. In particular, one has to explain the generation of a large (macroscopic) extra-dimension from an underlying theory.

3. Another prominent class of models of weak scale new physics is the Randall-Sundrum scenario [35]. It is based on a 5D space-time. The 5th dimension is compactified with an AdS-like geometry, and it has two boundaries. The particles in this model, including the SM, can localized toward either boundary or have a flat profile in between. This is a highly influential setup, and it has been instrumental in a huge amount of model building and phenomenological studies. For a lot of the RS constructions, the resulting phenomenology bears resemblance to that of the Composite Higgs models. This is not a surprise. AdS-CFT correspondence tells us that we should expect the physics of RS model can be captured by a 4D theory, and one of the boundaries of the RS bulk can be thought of as modeling the confinement in that 4D theory. The RS model, formulated as 5D warped compactification, provides a novel and transparent way of thinking about such physics.

4. One may feel that this line of attack focuses too much on eliminating the sensitivity to some high energy threshold. Why should this necessarily be the only possibility? We could imagine such a scenario that the parameters in the UV theory are just right to produce the low energy electroweak scale and associated physics. This can be enforced by some symmetry. However, in this case, the sensitivity to high energy threshold disappears, and it is not too different from Supersymmetry or other "usual" scenarios. It could happen through dynamics, because of some unusual UV/IR connection. This remains only as a logical possibility since we don't have a remotely workable scenario/framework which implements this idea. However, it would be very exciting if this is the case. It is much more dramatic than supersymmetry or any other new physics models we have dreamed of so far. This can happen by an accident, or by some kind of anthropic selection. Trying to find dynamical mechanism could be the wrong question to work on. We should certainly look for evidence for such an accident. However, until we find concrete evidence pointing to some other direction, our working assumption should be there is deep dynamical reason yet to be understood. Finally, it may not be as stupid as it looks to stick with the more "traditional" scenarios. Many of our more successful theories are based on some mechanism which makes it insensitive to the unknown UV physics. This includes the Big Bang Nucleosynthesis (by assuming thermal equilibrium in early universe) and inflation (by assuming an attractor in early universe). In addition, in many know cases of the Standard Model, theory is indeed natural in the way we expected. The prime example is again the low energy spectrum of the QCD.

To conclude our discussion on naturalness, I think it is still an extremely important and acute question in particle physics. The fact that we don't know the solution yet certainly does not mean that the question does not exist or can be dismissed. It is not from some abstract understanding of renormalization or divergence. It stems from the need of having a UV completion from which we can predict the weak scale. In this sense, it is well defined and unambiguous. Our current ideas can still work, it is crucial to test them further. On the other hand, we could need dramatically new 
ideas. This is exciting, even though we don't have even a vague idea where the new direction is yet.

\section{Suggestions for further study}

In this section, we suggest some reviews on various topics covered in this lecture. The list is certainly incomplete, it is intended to be starting places for further study.

1. Effective Field Theory. The Effective Field Theory methods have a lot of applications, with many recent and highly specialized developments. The basics are covered in these lecture notes [36].

2. The Standard Model. There are many excellent books covering the Standard Model [37]. There are also many lecture notes, and I have benefited particularly from these [38].

3. Flavor and $C P$. This is a huge topic with rich physics. Here are a couple of introductory reviews [39]. Neutrino masses and mixings are also subjects with a large body of literature. Here are a couple of reviews [40].

4. Dark Matter. It is an ever expanding field, limited only by our imagination. For the conventional WIMP story, there is this classic review [41]. Even though it focuses on the SUSY WIMPs, there is a large part of the review covering generic WIMP properties and detection methods, which is also applicable to other WIMP candidates. Strong CP problem and its associated solutions are reviewed in [42], focusing on the dynamical relaxation solution which leads to the existence of the axion. Axion physics is covered in [43].

5. Baryogenesis. There are many scenarios and models. The basics and the most popular models are reviewed here [44].

6. Cosmological Constant. It is a topic which can be confusing, also with many dead ends. These reviews [45] help to clarify a lot of the related issues.

7. SUSY phenomenology. Given that it is the favorite of most of the community for nearly two decades, there is a huge body of literature. Here, we only point to two of the most classic reviews [46].

8. Composite Higgs Composite Higgs also has a long history, it is enjoying a revival of interests in recent years. Here are two excellent reviews which also cover a lot of recent developments [47].

\section{References}

[1] S. L. Glashow, Nucl. Phys. 22, 579 (1961). doi:10.1016/0029-5582(61)90469-2. S. Weinberg, Phys. Rev. Lett. 19, 1264 (1967). doi:10.1103/PhysRevLett.19.1264. A. Salam, Conf. Proc. C 680519, 367 (1968). 
[2] P. W. Anderson, Phys. Rev. 130, 439 (1963). doi:10.1103/PhysRev.130.439. F. Englert and R. Brout, Phys. Rev. Lett. 13, 321 (1964). doi:10.1103/PhysRevLett.13.321. P. W. Higgs, Phys. Rev. Lett. 13, 508 (1964). doi:10.1103/PhysRevLett.13.508. G. S. Guralnik, C. R. Hagen and T. W. B. Kibble, Phys. Rev. Lett. 13, 585 (1964). doi:10.1103/PhysRevLett.13.585

[3] G. Aad et al. [ATLAS Collaboration], Phys. Lett. B 716, 1 (2012) doi:10.1016/j.physletb.2012.08.020 [arXiv:1207.7214 [hep-ex]]. S. Chatrchyan et al. [CMS Collaboration], Phys. Lett. B 716, 30 (2012) doi:10.1016/j.physletb.2012.08.021 [arXiv:1207.7235 [hep-ex]].

[4] J. M. Cornwall, D. N. Levin and G. Tiktopoulos, Phys. Rev. D 10, 1145 (1974) Erratum: [Phys. Rev. D 11, 972 (1975)]. doi:10.1103/PhysRevD.10.1145, 10.1103/PhysRevD.11.972. C. E. Vayonakis, Lett. Nuovo Cim. 17, 383 (1976). doi:10.1007/BF02746538

[5] M. S. Chanowitz and M. K. Gaillard, Nucl. Phys. B 261, 379 (1985). doi:10.1016/0550-3213(85)90580-2

[6] S. Weinberg, Phys. Rev. D 13, 974 (1976) Addendum: [Phys. Rev. D 19, 1277 (1979)]. doi:10.1103/PhysRevD.19.1277, 10.1103/PhysRevD.13.974 S. Dimopoulos and L. Susskind, Nucl. Phys. B 155, 237 (1979). doi:10.1016/0550-3213(79)90364-X

[7] D. J. Gross and F. Wilczek, Phys. Rev. Lett. 30, 1343 (1973). doi:10.1103/PhysRevLett.30.1343. H. D. Politzer, Phys. Rev. Lett. 30, 1346 (1973). doi:10.1103/PhysRevLett.30.1346

[8] L. Dixon, Lecture given at the TASI 2017 summer school.

[9] G. 't Hooft, NATO Sci. Ser. B 59, 135 (1980). doi:10.1007/978-1-4684-7571-59

[10] Y. Nambu, Phys. Rev. 117, 648 (1960). doi:10.1103/PhysRev.117.648. J. Goldstone, Nuovo Cim. 19, 154 (1961). doi:10.1007/BF02812722

[11] M. Gell-Mann, "The Eightfold Way: A Theory of strong interaction symmetry," CTSL-20, TID-12608. S. Okubo, Prog. Theor. Phys. 27, 949 (1962). doi:10.1143/PTP.27.949

[12] Particle data group. http://pdg.lbl.gov/

[13] E. Witten, Nucl. Phys. B 223, 422 (1983). doi:10.1016/0550-3213(83)90063-9

[14] J. Wess and B. Zumino, Phys. Lett. 37B, 95 (1971). doi:10.1016/0370-2693(71)90582-X

[15] P. Sikivie, L. Susskind, M. B. Voloshin and V. I. Zakharov, Nucl. Phys. B 173, 189 (1980). doi:10.1016/0550-3213(80)90214-X

[16] LEP Electroweak Working Group [ALEPH and CDF and D0 and DELPHI and L3 and OPAL and SLD Collaborations and LEP Electroweak Working Group and Tevatron Electroweak Working Group and SLD Electroweak and Heavy Flavour Groups], arXiv:1012.2367 [hep-ex].

[17] S. Weinberg, Phys. Rev. Lett. 43, 1566 (1979). doi:10.1103/PhysRevLett.43.1566

[18] P. Minkowski, Phys. Lett. 67B, 421 (1977). doi:10.1016/0370-2693(77)90435-X. M. Gell-Mann, P. Ramond and R. Slansky, Conf. Proc. C 790927 (1979) 315 [arXiv:1306.4669 [hep-th]].

T. Yanagida, Conf. Proc. C 7902131, 95 (1979). R. N. Mohapatra and G. Senjanovic, Phys. Rev. Lett. 44, 912 (1980). doi:10.1103/PhysRevLett.44.912. J. Schechter and J. W. F. Valle, Phys. Rev. D 22, 2227 (1980). doi:10.1103/PhysRevD.22.2227

[19] M. Fukugita and T. Yanagida, Phys. Lett. B 174, 45 (1986). doi:10.1016/0370-2693(86)91126-3

[20] N. Cabibbo, Phys. Rev. Lett. 10, 531 (1963). doi:10.1103/PhysRevLett.10.531. M. Kobayashi and T. Maskawa, Prog. Theor. Phys. 49, 652 (1973). doi:10.1143/PTP.49.652 
[21] L. Wolfenstein, Phys. Rev. Lett. 51, 1945 (1983). doi:10.1103/PhysRevLett.51.1945

[22] Unitarity Triangle Fit Collaboration. http://www.utfit.org/UTfit/

[23] S. L. Glashow, J. Iliopoulos and L. Maiani, Phys. Rev. D 2, 1285 (1970). doi:10.1103/PhysRevD.2.1285

[24] B. Pontecorvo, Sov. Phys. JETP 7, 172 (1958) [Zh. Eksp. Teor. Fiz. 34, 247 (1957)]. Z. Maki, M. Nakagawa and S. Sakata, Prog. Theor. Phys. 28, 870 (1962). doi:10.1143/PTP.28.870

[25] R. D. Peccei and H. R. Quinn, Phys. Rev. Lett. 38, 1440 (1977). doi:10.1103/PhysRevLett.38.1440. F. Wilczek, Phys. Rev. Lett. 40, 279 (1978). doi:10.1103/PhysRevLett.40.279

[26] A. G. Cohen, D. B. Kaplan and A. E. Nelson, Nucl. Phys. B 349, 727 (1991). doi:10.1016/0550-3213(91)90395-E

[27] C. D. Froggatt and H. B. Nielsen, Nucl. Phys. B 147, 277 (1979). doi:10.1016/0550-3213(79)90316-X

[28] S. Weinberg, Phys. Rev. Lett. 59, 2607 (1987). doi:10.1103/PhysRevLett.59.2607

[29] J. L. Gervais and B. Sakita, Nucl. Phys. B 34, 632 (1971). doi:10.1016/0550-3213(71)90351-8. Y. A. Golfand and E. P. Likhtman, JETP Lett. 13, 323 (1971) [Pisma Zh. Eksp. Teor. Fiz. 13, 452 (1971)]. P. Ramond, Phys. Rev. D 3, 2415 (1971). doi:10.1103/PhysRevD.3.2415. D. V. Volkov and V. P. Akulov, Phys. Lett. 46B, 109 (1973). doi:10.1016/0370-2693(73)90490-5. J. Wess and B. Zumino, Nucl. Phys. B 70, 39 (1974). doi:10.1016/0550-3213(74)90355-1

[30] S. Dimopoulos and H. Georgi, Nucl. Phys. B 193, 150 (1981). doi:10.1016/0550-3213(81)90522-8

[31] M. T. Grisaru, W. Siegel and M. Rocek, Nucl. Phys. B 159, 429 (1979). doi:10.1016/0550-3213(79)90344-4. N. Seiberg, Phys. Lett. B 318, 469 (1993) doi:10.1016/0370-2693(93)91541-T [hep-ph/9309335].

[32] H. E. Haber and R. Hempfling, Phys. Rev. Lett. 66, 1815 (1991). doi:10.1103/PhysRevLett.66.1815 Y. Okada, M. Yamaguchi and T. Yanagida, Phys. Lett. B 262, 54 (1991). doi:10.1016/0370-2693(91)90642-4. J. R. Ellis, G. Ridolfi and F. Zwirner, Phys. Lett. B 257, 83 (1991). doi:10.1016/0370-2693(91)90863-L

[33] D. B. Kaplan and H. Georgi, Phys. Lett. 136B, 183 (1984). doi:10.1016/0370-2693(84)91177-8

[34] N. Arkani-Hamed, S. Dimopoulos and G. R. Dvali, Phys. Lett. B 429, 263 (1998) doi:10.1016/S0370-2693(98)00466-3 [hep-ph/9803315].

[35] L. Randall and R. Sundrum, Phys. Rev. Lett. 83, 3370 (1999) doi:10.1103/PhysRevLett.83.3370 [hep-ph/9905221]. L. Randall and R. Sundrum, Phys. Rev. Lett. 83, 4690 (1999) doi:10.1103/PhysRevLett.83.4690 [hep-th/9906064].

[36] J. Polchinski, In *Boulder 1992, Proceedings, Recent directions in particle theory* 235-274, and Calif. Univ. Santa Barbara - NSF-ITP-92-132 (92,rec.Nov.) 39 p. (220633) Texas Univ. Austin UTTG-92-20 (92,rec.Nov.) 39 p [hep-th/9210046].

A. V. Manohar, "Effective field theories," Lect. Notes Phys. 479, 311 (1997) doi:10.1007/BFb0104294 [hep-ph/9606222].

D. B. Kaplan, "Effective field theories," nucl-th/9506035.

[37] G. L. Kane, "Modern Elementary Particle Physics," REDWOOD CITY, USA: ADDISON-WESLEY (1987) 344 P. (THE ADVANCED BOOK PROGRAM).

H. Georgi, "Weak Interactions and Modern Particle Theory,” Menlo Park, Usa: Benjamin/cummings ( 1984) $165 p$. 
P. Langacker, "The standard model and beyond," Boca Raton, USA: CRC Pr. (2010) 663 p J. F. Donoghue, E. Golowich and B. R. Holstein, "Dynamics of the standard model," Camb. Monogr. Part. Phys. Nucl. Phys. Cosmol. 2, 1 (1992) [Camb. Monogr. Part. Phys. Nucl. Phys. Cosmol. 35 (2014)]. doi:10.1017/CBO9780511524370.

[38] S. Willenbrock, "Symmetries of the standard model," hep-ph/0410370. W. Skiba, "Effective Field Theory and Precision Electroweak Measurements," doi:10.1142/97898143271830001 arXiv:1006.2142 [hep-ph].

R. Rattazzi, TASI 2015. https://physicslearning.colorado.edu/tasi/tasi_2015/tasi_2015.html

[39] Y. Nir, "CP violation in and beyond the standard model," hep-ph/9911321.

G. Isidori, Y. Nir and G. Perez, "Flavor Physics Constraints for Physics Beyond the Standard Model," Ann. Rev. Nucl. Part. Sci. 60, 355 (2010) doi:10.1146/annurev.nucl.012809.104534 [arXiv:1002.0900 [hep-ph]].

[40] M. C. Gonzalez-Garcia and Y. Nir, "Neutrino masses and mixing: Evidence and implications," Rev. Mod. Phys. 75, 345 (2003) doi:10.1103/RevModPhys.75.345 [hep-ph/0202058].

R. N. Mohapatra et al., “Theory of neutrinos: A White paper,” Rept. Prog. Phys. 70, 1757 (2007) doi:10.1088/0034-4885/70/11/R02 [hep-ph/0510213].

[41] G. Jungman, M. Kamionkowski and K. Griest, “Supersymmetric dark matter,” Phys. Rept. 267, 195 (1996) doi:10.1016/0370-1573(95)00058-5 [hep-ph/9506380].

[42] R. D. Peccei, Adv. Ser. Direct. High Energy Phys. 3, 503 (1989). doi:10.1142/97898145032800013

[43] J. E. Kim and G. Carosi, “Axions and the Strong CP Problem,” Rev. Mod. Phys. 82, 557 (2010) doi:10.1103/RevModPhys.82.557 [arXiv:0807.3125 [hep-ph]].

P. Sikivie, “Axion Cosmology,” Lect. Notes Phys. 741, 19 (2008) doi:10.1007/978-3-540-73518-22 [astro-ph/0610440].

J. E. Kim, "Light Pseudoscalars, Particle Physics and Cosmology," Phys. Rept. 150, 1 (1987). doi:10.1016/0370-1573(87)90017-2

[44] J. M. Cline, "Baryogenesis," hep-ph/0609145.

D. E. Morrissey and M. J. Ramsey-Musolf, "Electroweak baryogenesis," New J. Phys. 14, 125003

(2012) doi:10.1088/1367-2630/14/12/125003 [arXiv:1206.2942 [hep-ph]].

S. Davidson, E. Nardi and Y. Nir, “Leptogenesis,” Phys. Rept. 466, 105 (2008) doi:10.1016/j.physrep.2008.06.002 [arXiv:0802.2962 [hep-ph]].

[45] S. Weinberg, "The Cosmological constant problems," astro-ph/0005265.

J. Polchinski, “The Cosmological Constant and the String Landscape,” hep-th/0603249.

[46] S. P. Martin, “A Supersymmetry primer,” Adv. Ser. Direct. High Energy Phys. 21, 1 (2010) [Adv. Ser. Direct. High Energy Phys. 18, 1 (1998)] doi:10.1142/97898128396570001, 10.1142/97898143075050001 [hep-ph/9709356].

H. E. Haber and G. L. Kane, "The Search for Supersymmetry: Probing Physics Beyond the Standard Model,’ Phys. Rept. 117, 75 (1985). doi:10.1016/0370-1573(85)90051-1

[47] G. Panico and A. Wulzer, “The Composite Nambu-Goldstone Higgs," Lect. Notes Phys. 913, pp.1 (2016) doi:10.1007/978-3-319-22617-0 [arXiv:1506.01961 [hep-ph]].

R. Contino, “The Higgs as a Composite Nambu-Goldstone Boson,” doi:10.1142/97898143271830005 arXiv:1005.4269 [hep-ph]. 